\title{
Pion-nucleon scattering in a meson-exchange model
}

\author{
A. M. Gasparyan, ${ }^{1,2}$ J. Haidenbauer, ${ }^{1}$ C. Hanhart, ${ }^{1}$ and J. Speth ${ }^{1}$ \\ ${ }^{1}$ Institut für Kernphysik (Theorie), Forschungszentrum Jülich GmbH, D-52425 Jülich, Germany \\ ${ }^{2}$ Institute of Theoretical and Experimental Physics, 117259, B. Cheremushkinskaya 25, Moscow, Russia
}

(Received 16 July 2003; published 21 October 2003)

\begin{abstract}
The $\pi N$ interaction is studied within a meson-exchange model and in a coupled-channels approach which includes the channels $\pi N, \eta N$, as well as three effective $\pi \pi N$ channels, namely, $\rho N, \pi \Delta$, and $\sigma N$. Starting out from an earlier model of the Jülich group systematic improvements in the dynamics and in some technical aspects are introduced. With the new model an excellent quantitative reproduction of the $\pi N$ phase shifts and inelasticity parameters in the energy region up to $1.9 \mathrm{GeV}$ and for total angular momenta $J \leqslant 3 / 2$ is achieved. Simultaneously, good agreement with data for the total and differential $\pi N \rightarrow \eta N$ transition cross sections is obtained. The connection of the $\pi N$ dynamics in the $S_{11}$ partial wave with the reaction $\pi N \rightarrow \eta N$ is discussed.
\end{abstract}

DOI: 10.1103/PhysRevC.68.045207

PACS number(s): 14.20.Gk, 13.75.Gx, 11.80.Gw, 24.10.Eq

\section{INTRODUCTION}

The $\pi N$ interaction is interesting for several reasons. First, it is one of the main sources of information about the baryon spectrum. Thereby it serves as a doorway to the understanding of QCD in the nonperturbative regime, and especially of the confining mechanism, which is most important for binding a system of quarks into a hadron. For example, experimental information about the mass, width, and decay of baryon resonances serves as a testing ground for several models of the internal structure of the nucleon and its excited states. Most of this information is extracted from partial wave analyses of $\pi N$ scattering data [1-3].

The $\pi N$ interaction is also interesting by itself. The wealth of accurate data and the richness of structures shown by them provide an excellent but also challenging testing ground for any model description in terms of effective degrees of freedom, e.g., for chiral perturbation theory [4,5] but also for the more conventional meson-exchange picture [6-8].

Finally, the $\pi N$ interaction is an important ingredient in many other hadronic reactions and in particular for the meson production in nucleon-nucleon $(N N)$ collisions $[9,10]$. $\pi N$ rescattering is an essential mechanism in the reaction $N N \rightarrow N N \pi$ near threshold [11-13]. There are also strong indications that rescattering involving the $\pi N$ system plays an important, if not dominant role, in the production of the $\eta$ $[14-17]$ and $\omega$ mesons $[18,19]$ and even for the associated strangeness production $(N N \rightarrow N \Lambda K, N N \rightarrow N \Sigma K) \quad[20,21]$. Thus, model investigations of such production reactions require solid information about the corresponding elementary reactions such as $\pi N \rightarrow \eta N, \pi N \rightarrow \omega N, \pi N \rightarrow K \Lambda, \pi N \rightarrow K \Sigma$, etc.

Over the past few years, in a series of papers, the Jülich group has investigated the $\pi N$ interaction in the mesonexchange framework $[8,22-24]$. One of the main novelties of the model was treating the $\sigma$ - and $\rho$-meson $t$-channel exchanges as correlated two-pion exchange, using the dispersion relation technique. The Jülich model was originally constructed to describe elastic $\pi N$ data not far from threshold [22]. Later the model was extended to higher energies by including several inelastic channels, namely, three effective $\pi \pi N$ channels $(\sigma N, \rho N$, and $\Delta \pi)$ and the $\eta N$ channel $[8,24]$. The treatment of correlated $\pi \pi$ exchange was made more consistent and transparent in Ref. [25]. The possibility of generating resonances dynamically was also systematically studied. It turned out that only one of them, namely the Roper resonance $P_{11}(1440)$, can be understood in this way in the framework of the Jülich $\pi N$ model [8,24]. Other resonances such as $S_{11}(1535), S_{11}(1650), D_{13}(1520)$, and $\Delta(1232)$ had to be included explicitly. The latest model provided a good qualitative, and in many partial waves even a quantitative, description of $\pi N$ scattering in the energy region from threshold up to $1.9 \mathrm{GeV}$. [8].

Unfortunately, a further improvement of this model by simply introducing further resonances and by including additional inelastic channels proved to be impossible due to several reasons. First of all, in some partial waves the deviation of the model predictions from the data at higher energies are seemingly not due to missing resonance contributions only. Already the basic (nonresonant or background) contributions of the model by Krehl et al. [8] are incompatible with the general trend exhibited by the experimental phase shifts.

The second problem is a strong influence of the $N^{*}(1650)$ resonance on the low energy $S_{11}$ phase shift. In fact, it gives the main contribution to this partial wave even at threshold which is, of course, unphysical. This means, in turn, that any additional channels that couple to the $N^{*}(1650)$ resonance will likewise have a strong influence on the $S_{11}$ phase shift close to threshold, a certainly undesirable feature.

Finally, the existing $\pi N$ model yields only an unsatisfactory description of the inelasticity parameter in the $S_{11}$ partial wave and at the same time it overestimates the $\pi N \rightarrow \eta N$ transition cross section close to the $\eta N$ threshold. These two related problems are believed to be due to shortcomings in the treatment of the $\pi \pi N$ channel.

In this context, let us mention that the $S_{11}$ partial wave is of particular importance for the $\eta N$ and $K \Lambda$ channels close to their thresholds. For $\pi N \rightarrow \eta N$ as well as $\pi N \rightarrow K \Lambda$ experimental information on the transition cross sections and also differential cross sections and polarization observables are available. An analysis of these data within our model re- 
quires a satisfactory description of the $S_{11} \pi N$ partial wave in the relevant energy range. Moreover, an adequate description of the $S_{11}$ inelasticity and of the $\pi N \rightarrow \eta N$ transition amplitude is also needed if one wishes to investigate $\eta$ production in $N N$ collisions [17]. Similarly, the $\pi N \rightarrow K \Lambda(\Sigma)$ transition amplitude plays an important role in studies of $\Lambda(\Sigma)$ production in $N N$ collisions. It is the main ingredient in the production amplitude based on the pion rescattering mechanism [20].

In the present work we want to remedy the abovementioned deficiencies of the Jülich $\pi N$ model [8]. Thereby we aim at a quantitative description of the $\pi N$ phase shifts and inelasticities for all partial waves with $J \leqslant 3 / 2$, from threshold up to around $1.9 \mathrm{Gev}$. A further and equally important goal is the consistent description of the experimental information on the $\pi N \rightarrow \eta N$ transition.

The paper is structured in the following way. In Sec. II the main ingredients of our $\pi N$ model are described with special emphasis on those parts of the dynamics where changes and improvements were made. For the time being, apart from the $\pi \pi N$ channel (described effectively via the $\sigma N, \rho N$, and $\Delta \pi$ channels) only the $\eta N$ channel is taken into account. However, the inclusion of the $K \Lambda$ channel (and even $\omega N$ and $K \Sigma$ ) is expected to be straightforward within the new improved model. In Sec. III we present results for the $\pi N$ elastic scattering. Specifically, we compare the $\pi N$ phase shifts and inelasticities of the new model with experimental values and with the description achieved within the model of Krehl et al. [8]. In addition, and as the main result of our paper we examine in detail the transition reaction $\pi N \rightarrow \eta N$. Calculations for the total transition cross section but also for differential observables are presented. Furthermore, we shed some light on peculiar structures which occur in the $\pi N \rightarrow \eta N$ total cross section of our old model, but also in other models in the literature [26-28]. The paper ends with a summary.

\section{DESCRIPTION OF THE MODEL}

The general framework as well as all technical aspects of the Jülich $\pi N$ model have been thoroughly described in earlier papers $[8,22,25]$. Therefore, we refrain from repeating all the details here. Rather we want to give a brief account of its main features with specific emphasis on the new and improved ingredients of the present model.

Our model of the $\pi N$ interaction is derived within the meson-exchange framework in time-ordered perturbation theory. Within the envisaged range of validity of our model of up to around $1.9 \mathrm{GeV}$ inelasticities play an increasingly important role, as is evidenced by the results of phase-shift analyses. Hence, coupling to reaction channels that are responsible for these inelasticities have to be taken into account. The decay modes of the nucleon resonances in the energy range under consideration show that the dominant decay [besides $\pi N$ and $\eta N$ for the $N^{*}(1535)$ ] is the $\pi \pi N$ channel [29]. Since a three-body calculation is much too complicated for realistic potentials, we represent the $\pi \pi N$ channel by effective two-body channels. In doing this we are guided by studying strong interactions, between two-body clusters of the three-body $\pi \pi N$ state in the spirit of the for- malism of Ref. [30]. The dominant clusters are the $\Delta$ in the $\pi N$ interaction, the $\rho$ in the vector-isovector $\pi \pi$ interaction and the strong correlation in the scalar-isoscalar $\pi \pi$ interaction, which we call $\sigma$. Therefore-besides the $\pi N$ and $\eta N$ channels-we include in our model the reaction channels $\pi \Delta, \sigma N$, and $\rho N$.

Accordingly, we have to solve the coupled-channel scattering equation [31]

$$
\begin{aligned}
\left\langle\vec{k}^{\prime} \lambda_{3} \lambda_{4}\left|T_{\mu \nu}^{I}\right| \vec{k} \lambda_{1} \lambda_{2}\right\rangle= & \left\langle\vec{k}^{\prime} \lambda_{3} \lambda_{4}\left|V_{\mu \nu}^{I}\right| \vec{k} \lambda_{1} \lambda_{2}\right\rangle \\
& +\sum_{\gamma} \sum_{\lambda_{1}^{\prime}, \lambda_{2}^{\prime}} \int d^{3} q\left\langle\vec{k}^{\prime} \lambda_{3} \lambda_{4}\left|V_{\mu \gamma}^{I}\right| \vec{q} \lambda_{1} \lambda^{\prime}\right\rangle \\
& \times \frac{1}{E-W_{\gamma}(q)+i \epsilon}\left\langle\vec{q} \lambda_{1}^{\prime} \lambda_{2}^{\prime}\left|T_{\gamma \nu}^{I}\right| \vec{k} \lambda_{1} \lambda_{2}\right\rangle,
\end{aligned}
$$

where $\lambda_{i}, \lambda_{i+2}, \lambda_{i}^{\prime},(i=1,2)$ are the helicities of the baryon and meson in the initial, final, and intermediate states, $I$ is the total isospin of the two body system, and $\mu, \nu, \gamma$ are indices that label different reaction channels. $W_{\gamma}(q)$ $=\sqrt{q^{2}+M_{\gamma}}+\sqrt{q^{2}+m_{\gamma}}$, where $m_{\gamma}\left(M_{\gamma}\right)$ is the mass of the meson (baryon) in the channel $\gamma$. We work in the center-ofmomentum frame and $k\left(k^{\prime}\right)$ are the momenta of the initial (final) baryon.

The pseudopotential $V_{\mu \nu}^{I}$ (i.e., the interaction between baryon and meson) that is iterated in Eq. (1) is constructed from an effective Lagrangian. Our interaction Lagrangian (see Table I) is based on that of Wess and Zumino [32], which we have supplemented with additional terms for including the $\Delta$ isobar, the $\omega, \eta, a_{0}$ meson, and the $\sigma$. We also have included terms that characterize the coupling of the resonances $N^{*}(1535), N^{*}(1520)$, and $N^{*}(1650)$ to various reaction channels. The diagrams that built up the interaction in the $\pi N \rightarrow \pi N, \pi N \rightarrow \eta N$, and $\eta N \rightarrow \eta N$ channels are shown in Figs. 1 and 2 as an example and also to introduce our notation. The full set of diagrams, including also the transitions and interactions in the other reaction channels $(\rho N, \sigma N$, and $\pi \Delta$ ), can be found in Ref. [8]. In that paper one can also find explicit expressions for all the matrix elements $\left\langle\overrightarrow{k^{\prime}} \lambda_{3} \lambda_{4}\left|V_{\mu \nu}^{I}\right| \vec{k} \lambda_{1} \lambda_{2}\right\rangle$.

As already indicated in the Introduction, there are some modifications and improvements in the present model and we want to summarize them here. First, we now use derivative coupling for the $S_{11} N^{*}$ resonances, as demanded by chiral symmetry. The corresponding Lagrangians for the $N^{*}\left(S_{11}\right) N \pi$ and $N^{*}\left(S_{11}\right) N \eta$ vertices can be found in Table I. Second, we introduce a coupling of the $S_{11} N^{*}(1535)$ resonance to the $\pi \Delta$ channel. Also, this Lagrangian is given in Table I. Finally, the subtraction constant that appears in the dispersion relations which constitute the contribution of the correlated $\pi \pi$ exchange in the scalar-isoscalar $(\sigma)$ channel [22] is not set to zero as in our previous models [8,24], but allowed to assume a finite value. Interpreted in terms of effective exchanges this contact term corresponds to the exchange of a $\sigma$ meson with scalar coupling in addition to the derivative coupling as it occurs now for the $\sigma$ exchange stemming from the subtracted dispersion integral. Note, 
TABLE I. The effective Lagrangian.

\begin{tabular}{|c|c|c|c|}
\hline Vertex & $\mathcal{L}_{i n t}$ & Vertex & $\mathcal{L}_{i n t}$ \\
\hline$N N \pi$ & $-\frac{f_{N N \pi}}{m_{\pi}} \bar{\Psi} \gamma^{5} \gamma^{\mu} \vec{\tau} \partial_{\mu} \vec{\pi} \Psi$ & $\pi \eta a_{0}$ & $g_{\pi \eta a_{0}} m_{\pi} \eta \vec{\pi} \vec{a}_{0}$ \\
\hline$N \Delta \pi$ & $\frac{f_{N \Delta \pi}}{m_{\pi}} \bar{\Delta}^{\mu} \vec{S}^{\dagger} \partial_{\mu} \vec{\pi} \Psi+$ H.c. & $N^{*}\left(S_{11}\right) N \pi$ & $\frac{f_{N^{*} N \pi}}{m_{\pi}} \bar{\Psi}_{N^{*}} \gamma^{\mu} \vec{\tau} \Psi \partial_{\mu} \vec{\pi}+$ H.c. \\
\hline$\rho \pi \pi$ & $-g_{\rho \pi \pi}\left(\vec{\pi} \times \partial_{\mu} \vec{\pi}\right) \vec{\rho}^{\mu}$ & $N^{*}\left(S_{11}\right) N \eta$ & $\frac{f_{N^{*} N \eta}}{m_{\pi}} \bar{\Psi}_{N^{*}} \gamma^{\mu} \Psi \partial_{\mu} \eta+$ H.c. \\
\hline$N N \rho$ & $-g_{N N \rho} \bar{\Psi}\left[\gamma^{\mu}-\frac{\kappa_{\rho}}{2 m_{N}} \sigma^{\mu \nu} \partial_{\nu}\right] \vec{\tau} \vec{\rho}_{\mu} \Psi$ & $N^{*}\left(S_{11}\right) N \rho$ & $g_{N^{*} N \rho} \bar{\Psi}_{N^{*}} \gamma^{5} \gamma^{\mu} \vec{\tau}_{\rho_{\mu}} \Psi+$ H.c. \\
\hline$N N \sigma$ & $-g_{N N \sigma} \bar{\Psi} \Psi \sigma$ & $N^{*}\left(S_{11}\right) \Delta \pi$ & $\frac{-f_{N^{*} \Delta \pi}}{m_{\pi}} \Psi_{N^{*}} \gamma^{5} \vec{S} \Delta^{\mu} \partial_{\mu} \vec{\pi}+$ H.c. \\
\hline$\sigma \pi \pi$ & $\frac{g_{\sigma \pi \pi}}{2 m_{\pi}} \partial_{\mu} \vec{\pi} \partial^{\mu} \vec{\pi} \sigma$ & $N^{*}\left(P_{13}\right) N \pi$ & $\frac{f_{N^{*} N \pi}}{m_{\pi}} \Psi_{N^{*}}^{\mu} \vec{\tau} \Psi \partial_{\mu} \vec{\pi}+$ H.c. \\
\hline$\sigma \sigma \sigma$ & $-g_{\sigma \sigma \sigma} m_{\sigma} \sigma \sigma \sigma$ & $N^{*}\left(P_{13}\right) N \eta$ & $\frac{f_{N^{*} N \eta}}{m_{\pi}} \Psi_{N^{*}}^{\mu} \Psi \partial_{\mu} \eta+$ H.c. \\
\hline$N N \rho \pi$ & $\frac{f_{N N \pi}}{m_{\pi}} g_{\rho} \bar{\Psi} \gamma^{5} \gamma^{\mu} \vec{\tau} \Psi\left(\vec{\rho}_{\mu} \times \vec{\pi}\right)$ & $N^{*}\left(P_{13}\right) \Delta \pi$ & $\frac{f_{N^{*} \Delta \pi}}{m_{\pi}} \Psi_{N^{*}}^{\mu} \gamma^{5} \gamma^{\nu} \vec{S} \Delta_{\mu} \partial_{\nu} \vec{\pi}+$ H.c. \\
\hline$N N a_{1}$ & $-\frac{f_{N N \pi}}{m_{\pi}} m_{a_{1}} \bar{\Psi} \gamma^{5} \gamma^{\mu} \vec{\tau} \Psi \vec{a}_{\mu}$ & $N^{*}\left(D_{13}\right) N \pi$ & $\frac{f_{N^{*} N \pi}}{m_{\pi}^{2}} \bar{\Psi} \gamma^{5} \gamma^{\nu} \vec{\tau} \Psi_{N^{*}}^{\mu} \partial_{\nu} \partial_{\mu} \vec{\pi}+$ H.c. \\
\hline$a_{1} \pi \rho$ & $\begin{array}{l}-\frac{g_{\rho}}{m_{a_{1}}}\left[\partial_{\mu} \vec{\pi} \times \vec{a}_{\nu}-\partial_{\nu} \vec{\pi} \times \vec{a}_{\mu}\right]\left[\partial^{\mu} \vec{\rho}^{\nu}-\partial^{\nu} \vec{\rho}^{\mu}\right] \\
+\frac{g_{\rho}}{2 m_{a_{1}}}\left[\vec{\pi} \times\left(\partial_{\mu} \vec{\rho}_{\nu^{-}}-\partial_{\nu} \vec{\rho}_{\mu}\right)\right]\left[\partial^{\mu} \vec{a}^{\nu}-\partial^{\nu} \vec{a}^{\mu}\right]\end{array}$ & $N^{*}\left(D_{13}\right) N \eta$ & $\frac{f_{N^{*} N \eta}}{m_{\pi}^{2}} \bar{\Psi} \gamma^{5} \gamma^{\nu} \Psi_{N^{*}}^{\mu} \partial_{\nu} \partial_{\mu} \eta+$ H.c. \\
\hline$N N \omega$ & $-g_{N N \omega} \bar{\Psi} \gamma^{\mu} \omega_{\mu} \Psi$ & $N^{*}\left(D_{13}\right) \Delta \pi$ & $i \frac{f_{N^{*} \Delta \pi}}{m_{\pi}} \bar{\Psi}_{N^{*}{ }_{\nu}} \vec{S} \gamma^{\mu} \Delta^{\nu} \partial_{\mu} \vec{\pi}+$ H.c. \\
\hline$\omega \pi \rho$ & $\frac{g_{\omega \pi \rho}}{m_{\omega}} \epsilon_{\alpha \beta \mu \nu} \partial^{\alpha} \vec{\rho}^{\beta} \partial^{\mu} \vec{\pi} \omega^{\nu}$ & $N^{*}\left(D_{13}\right) N \rho$ & $\frac{f_{N^{*} N \rho}}{m_{\rho}} \bar{\Psi}_{N^{*}}^{\mu} \gamma^{\nu} \vec{\tau} \vec{\rho}_{\mu \nu} \Psi+$ H.c. \\
\hline$N \Delta \rho$ & $-i \frac{f_{N \Delta \rho}}{m_{\rho}} \bar{\Delta}^{\mu} \gamma^{5} \gamma^{\nu} \vec{S}^{\dagger} \vec{\rho}_{\mu \nu} \Psi+$ H.c. & $\Delta^{*}\left(S_{31}\right) N \pi$ & $\frac{f_{\Delta^{*} N \pi}}{m_{\pi}} \bar{\Delta}^{*} \gamma^{\mu} \vec{S}^{\dagger} \Psi \partial_{\mu} \vec{\pi}+$ H.c. \\
\hline$\rho \rho \rho$ & $\frac{g_{\rho}}{2}\left(\vec{\rho}_{\mu} \times \vec{\rho}_{\nu}\right) \vec{\rho}^{\mu \nu}$ & $\Delta^{*}\left(S_{31}\right) \Delta \pi$ & $\frac{-f_{\Delta^{*} \Delta \pi}}{m_{\pi}} \bar{\Delta}^{*} \gamma^{5} \vec{T} \Delta^{\mu} \partial_{\mu} \vec{\pi}+$ H.c. \\
\hline$N N \rho \rho$ & $\frac{\kappa_{\rho} g_{\rho}^{2}}{8 m_{N}} \bar{\Psi} \sigma^{\mu \nu} \vec{\tau} \Psi\left(\vec{\rho}_{\mu} \times \vec{\rho}_{\nu}\right)$ & $\Delta^{*}\left(P_{31}\right) N \pi$ & $-\frac{f_{\Delta^{*} N \pi}}{m_{\pi}} \bar{\Delta}^{*} \gamma^{5} \gamma^{\mu} \vec{S}^{\dagger} \Psi \partial_{\mu} \vec{\pi}+$ H.c. \\
\hline$\Delta \Delta \pi$ & $\frac{f_{\Delta \Delta \pi}}{m_{\pi}} \bar{\Delta}_{\mu} \gamma^{5} \gamma^{\nu} \vec{T} \Delta^{\mu} \partial_{\nu} \vec{\pi}$ & $\Delta^{*}\left(P_{31}\right) \Delta \pi$ & $-\frac{f_{\Delta^{*} \Delta \pi}}{m_{\pi}} \bar{\Delta}^{*} \vec{T} \Delta^{\mu} \partial_{\mu} \vec{\pi}+$ H.c. \\
\hline$\Delta \Delta \rho$ & $-g_{\Delta \Delta \rho} \bar{\Delta}_{\tau}\left(\gamma^{\mu}-i \frac{\kappa_{\Delta \Delta \rho}}{2 m_{\Delta}} \sigma^{\mu \nu} \partial_{\nu}\right) \vec{\rho}_{\mu} \vec{T} \Delta^{\tau}$ & $N^{*}\left(D_{33}\right) N \pi$ & $\frac{f_{N^{*} N \pi}}{m_{\pi}^{2}} \bar{\Psi} \gamma^{5} \gamma^{\nu} \vec{S}^{\dagger} \Psi_{N^{*}}^{\mu} \partial_{\nu} \partial_{\mu} \vec{\pi}+$ H.c. \\
\hline$N N \eta$ & $-\frac{f_{N N \eta}}{m_{\pi}} \bar{\Psi} \gamma^{5} \gamma^{\mu} \partial_{\mu} \eta \Psi$ & $N^{*}\left(D_{33}\right) \Delta \pi$ & $i \frac{f_{N^{*} \Delta \pi}}{m_{\pi}} \bar{\Delta}^{*} \nu \vec{T} \gamma^{\mu} \Delta^{\nu} \partial_{\mu} \vec{\pi}+$ H.c. \\
\hline$N N a_{0}$ & $g_{N N a_{0}} m_{\pi} \bar{\Psi} \vec{\tau} \Psi \vec{a}_{0}$ & $N^{*}\left(D_{33}\right) N \rho$ & $\frac{f_{N^{*} N \rho}}{m_{\rho}} \bar{\Delta}^{*} \mu \gamma^{\nu} \vec{S}^{\dagger} \vec{\rho}_{\mu \nu} \Psi+$ H.c. \\
\hline
\end{tabular}




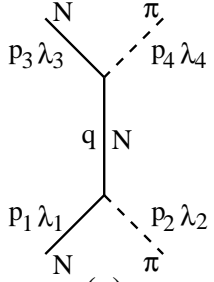

(a)

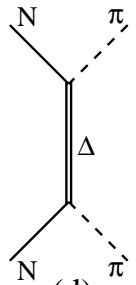

(d)

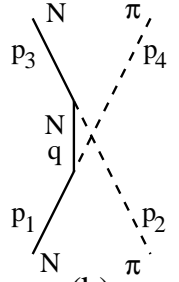

(b)

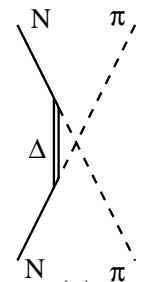

(e)

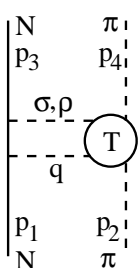

(c)

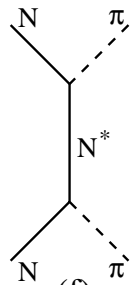

(f)
FIG. 1. Contribution to the elastic $\pi N$ channel.

when interpreting the low energy constants $c_{i}$, as they occur in the chiral perturbation theory analysis of $\pi N$ scattering, phenomenologically in terms of resonance exchanges, both coupling structures of a scalar to pions can also be identified [33]. Explicit expressions for those matrix elements $\left\langle\vec{k}^{\prime} \lambda_{3} \lambda_{4}\left|V_{\mu \nu}^{I}\right| \vec{k} \lambda_{1} \lambda_{2}\right\rangle$ which differ from the ones employed in our old model [8] can be found in the Appendix.

Mesons and baryons are not pointlike particles, but have a finite size. Therefore the interaction vertices $\mathrm{mmm}$ and $\mathrm{mBB}$ ( $m$ is meson, $B$ is baryon) also have a finite structure which, in our model, is taken into account by means of form factors. These form factors are parametrized by the following analytical forms, in which $\vec{q}$ is the three-momentum transfer carried by the exchanged particle.

For meson and baryon exchange

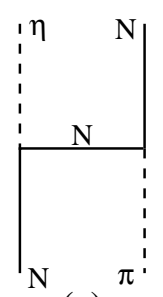

(a)

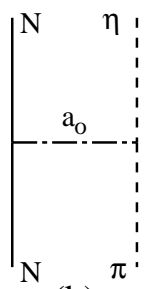

(b)

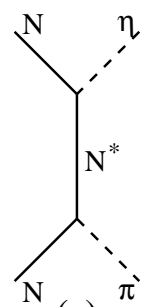

(c)

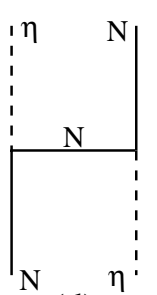

(d)

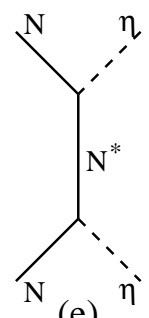

(e)
FIG. 2. Contribution to the $\pi N \rightarrow \eta N$ transition (a)-(c) and to the $\eta N$ channel (d)-(e).

$$
F(q)=\left(\frac{\Lambda^{2}-m_{x}^{2}}{\Lambda^{2}+\vec{q}^{2}}\right)^{n}
$$

We use monopole form factors $(n=1)$ except for the $\Delta$ exchange, for which the convergence of the integral in Eq.

(1) requires a dipole form factor $(n=2)$.

For the nucleon exchange at the $\pi N N$ vertex

$$
F(q)=\frac{\Lambda^{2}-m_{N}^{2}}{\Lambda^{2}-\left[\left(m_{N}^{2}-m_{\pi}^{2}\right) / m_{N}\right]^{2}+\vec{q}^{2}} .
$$

This choice ensures that the nucleon pole and nucleon exchange contribution cancel each other at the ChengDashen point, which is needed for a calculation of the $\Sigma$ term [22].

For $N, N^{*}$, and $\Delta$ pole diagrams

$$
F(q)=\left(\frac{\Lambda^{4}+m_{R}^{4}}{\Lambda^{4}+\left[E_{\gamma}(q)+\omega_{\gamma}(q)\right]^{4}}\right)^{n},
$$

where $n=1$ is used for $S$ - and $P$-wave resonances, and $n$ $=2$ for resonances in higer partial waves.

The correlated $\pi \pi$ exchange is supplemented by the form factor

$$
F\left(p_{2}, p_{4}\right)=\frac{\Lambda^{2}}{\Lambda^{2}+\vec{p}_{2}^{2}} \frac{\Lambda^{2}}{\Lambda^{2}+\vec{p}_{4}^{2}}
$$

Note that this choice differs from the form employed in our previous $\pi N$ models, where the form factor appeared inside the $t^{\prime}$ integration, cf. Ref. [22]. The particular form we apply in the present work has the following advantages: (i) it does not depend on energy; (ii) it does not modify strongly the on-shell potential (which is assumed to be fully determined by the dispersion integrals) as long as the energy is not too high; and (iii) it does not change the $t$ dependence of the potential.

For the contact interaction in the Wess-Zumino Lagrangian [32],

$$
F\left(p_{2}, p_{4}\right)=\left(\frac{\Lambda^{2}+m_{4}^{2}}{\Lambda^{2}+\vec{p}_{4}^{2}} \frac{\Lambda^{2}+m_{2}^{2}}{\Lambda^{2}+\vec{p}_{2}^{2}}\right)^{2} .
$$

Finally, we want to emphasize that the $\Delta$ isobar in the $\pi \Delta$ channel and the $\sigma$ and $\rho$ mesons in the $\sigma N$ and $\rho N$ channels are not treated as stable particles. Rather, as already mentioned above, the $\Delta, \sigma$, and $\rho$ here stand for $\pi N$ and $\pi \pi$ subsystems with the quantum numbers of the $P_{33}$ partial wave in the $\pi N$ system and the $I=J=0$ and $I=J=1$ partial waves in the $\pi \pi$ system, respectively. In order to simulate these, a simplified model for the $P_{33} \pi N$ partial wave as well as for the $\delta_{00}$ and $\delta_{11} \pi \pi$ partial waves was adopted in which pole diagrams, in the framework of time-ordered perturbation theory, are iterated [8,24]. These models are then used to construct the self-energies of the $\Delta, \sigma$, and $\rho$ which appear in the propagators of the $\pi \Delta$ and $\sigma N$ intermediate states in our scattering equation, i.e., we replace the two-particle intermediate state propagator for $\pi \Delta, \sigma N$, and $\rho N$ by 
TABLE II. Masses of mesons and baryons (in $\mathrm{MeV}$ ) used in the calculations.

\begin{tabular}{cccccc}
\hline \hline \multicolumn{3}{c}{ Mesons } & \multicolumn{2}{c}{ Baryons } \\
\hline$m_{\pi}$ & 138.03 & $m_{\omega}$ & 782.6 & $m_{N}$ & 938.926 \\
$m_{\eta}$ & 547.45 & $m_{a_{0}}$ & 982.7 & $m_{\Delta}$ & 1232.0 \\
$m_{\sigma}$ & 650.0 & $m_{a_{1}}$ & 1260.0 & & \\
$m_{\rho}$ & 769.0 & & & & \\
\hline \hline
\end{tabular}

$$
\frac{1}{E-W_{\gamma}(q)} \rightarrow \frac{1}{E-W_{\gamma}(q)-\Sigma_{\gamma}\left(E_{s u b}\right)}
$$

where

$$
\begin{aligned}
& E_{\text {sub }}=E-\omega_{\pi}(q)-\left[\sqrt{\left(M_{\Delta}^{o}\right)^{2}+\vec{q}^{2}}-M_{\Delta}^{o}\right] \text { for the } \Delta, \\
& E_{\text {sub }}=E-E_{N}(q)-\left[\sqrt{\left(m_{r}^{o}\right)^{2}+\vec{q}^{2}}-m_{r}^{o}\right] \text { for } r=\rho, \sigma
\end{aligned}
$$

is the energy of the decaying cluster at rest [24]. The bare masses $M_{\Delta}^{o}$ and $m_{r}^{o}$ are determined by fitting the models to the relevant phase shifts of the $\pi N$ and $\pi \pi$ systems, cf. Refs. [8,24] for details. By taking into account the selfenergy contibutions we preserve the correct threshold behavior for the description of pion production in the $\pi N$ system.

The scattering equation (1) is reduced to a set of onedimensional integral equations by means of the usual partial wave decomposition [34] and then solved numerically by standard contour-deformation methods [35,36].

\section{RESULTS}

In this section we present the results of our model for $\pi N$ elastic scattering and for the $\pi N \rightarrow \pi \eta$ transition in the energy range from $\pi N$ threshold up to $1.9 \mathrm{GeV}$. First we discuss the parameters that enter into our model calculation. Then we present the results for the $\pi N$ phase shifts and inelasticities. In particular, the role of the background and of the resonance contributions is analyzed. We also compare the results with those of the previous version of the model. Furthermore, we analyze in detail the results for the $\pi N \rightarrow \eta N$ total cross section and angular distributions and discuss the role of the background in this process.

\section{A. Parameters of the model}

Our model is based on the effective potential, which was described in Sec. II. The masses of all the particles appearing in the model are collected in Table II. Here one should pay attention to the mass of the $\sigma$ meson. While the $\sigma$ exchange in the $\pi N \rightarrow \pi N$ potential is evaluated using a dispersion relation, we have another $t$-channel $\sigma$ exchange in the $\sigma N$ $\rightarrow \sigma N$ potential. In this case we choose the value $m_{\sigma}$ $=650 \mathrm{MeV}$, which was extracted from a Breit-Wigner parametrization of the correlated $\pi \pi$ exchange in Ref. [37].

Table III contains coupling constants and cutoff parameters of the form factors for the vertices entering the $t$ - and $u$-exchange diagrams and the contact terms, i.e., those which constitute the background.
Most of the coupling constants have been taken from other sources. The coupling constants of the pole diagrams are constrained by values determined from their decay widths, for which we take the estimates of Ref. [38]. The parameters which are not fixed from other sources are shown in boldface. These are the purely phenomenological coupling constant at the triple $\sigma$ vertex $g_{\sigma \sigma \sigma}$ and the subtraction constant $A_{0}$ for the dispersion relation in the $\sigma$ channel. In addition, the cutoff masses are treated as free parameters. These free parameters are determined by a fit to the $\pi N$ phase shifts and inelasticities for $J \leqslant 3 / 2$ and the $\pi N \rightarrow \eta N$ cross section in the energy range from threshold to about $1.9 \mathrm{GeV}$. Here we should emphasize that we restrict ourselves to values of the cutoff masses of about $1-1.5 \mathrm{GeV}$ (in some cases up to $2 \mathrm{GeV}$ for heavy exchanged particles), i.e., values in line with typical hadronic scales.

Parameters of the pole diagrams (bare masses and coupling constants) are given in Table IV. Note that the bare nucleon mass and bare $\pi N$ coupling constant $f_{\pi N N}^{B}$ are not free parameters, because they are fixed by the physical values of these quantities (cf. Ref. [22]). However, the cutoff at the $\pi N N$ vertex was allowed to vary, in order to fit the $P_{11}$ partial wave. The resulting parameters for the nucleon pole are

$$
M_{0}=1239 \mathrm{MeV}, \frac{\left(f_{N N \pi}^{B}\right)^{2}}{4 \pi}=0.0166, \Lambda=1950 \mathrm{MeV}
$$

The cutoff masses for all other resonance diagrams were set to $2 \mathrm{GeV}$. Indeed, the results depend only weakly on the particular values of the cutoff masses, since their effects can be always compensated by a change in the corresponding coupling constants. The largeness of the cutoff masses in the resonance diagrams is motivated by the specific analytical form of the employed resonance form factors, which fall off with momentum rather rapidly even for such a large cutoff mass [39].

In general, we adopt positive values for the sign of the bare coupling constants. However, we use negative coupling constants if this leads to a better agreement with the data. In the case of the $N_{P_{13}}^{*}(1720) \eta N$ vertex we changed the sign of the coupling constant because this allows us to obtain a better description of the $\pi N \rightarrow \eta N$ differential cross section via an interference of the $P_{13}$ with other partial waves. Finally, we would like to remark that among the three phase-shift analyses, whose results are shown in figures, we use the energy independent analysis from Ref. [2] as the main guideline for the fitting procedure.

\section{B. $\pi N$ elastic scattering}

We start the discussion of the elastic $\pi N$ data by first looking at the phase shifts as they result from the original model of Krehl et al. [8] (cf. the dashed curves in Figs. 3, 4, and 5) In general, the quality of the description is rather good, but there are some unsatisfactory features which we would like to point out here.

First, there are significant deviations of the model results from the data in some partial waves, specifically in the $P_{13}$, $S_{31}$, and $D_{33}$ waves. Evidently, the discrepancies are prima- 
TABLE III. Parameters of the vertices which enter into the background diagrams. Free parameters are given in boldface.

\begin{tabular}{|c|c|c|c|c|}
\hline Vertex & Type of the diagram & Coupling constant & Reference & Cutoff $\Lambda(\mathrm{MeV})$ \\
\hline \multicolumn{5}{|c|}{ Correlated $\pi \pi$ exchange: } \\
\hline & $\rho$ channel & & & 1000 \\
\hline & $\sigma$ channel & $A_{0}=\mathbf{2 5} \mathrm{MeV} / \mathrm{F}_{\pi}^{2}$ & & 900 \\
\hline$N N \pi$ & $N$ exchange & $\frac{f_{N N \pi}^{2}}{4 \pi}=0.0778$ & {$[59]$} & 1100 \\
\hline$N \Delta \pi$ & $\Delta$ exchange & $\frac{f_{N \Delta \pi}^{2}}{4 \pi}=0.36$ & [59] & 1800 \\
\hline$N N \rho$ & $N$ exchange & $\frac{g_{N N \rho}^{2 \prime \prime}}{4 \pi}=0.84$ & $\begin{array}{l}{[59]} \\
{[59]}\end{array}$ & 1600 \\
\hline$N N \rho \pi$ & Contact term & $\sim f_{N N \pi} g_{N N \rho}$ & & 1100 \\
\hline$N N \pi$ & $\pi$ exchange & $\sim f_{N N \pi}$ & & 900 \\
\hline$\pi \pi \rho$ & $\pi$ exchange & $\frac{g_{\pi \pi \rho}^{2}}{4 \pi}$ & & 900 \\
\hline$N N \omega$ & $\omega$ exchange & $\frac{g_{N N \omega}^{2}}{4 \pi}=11.0$ & [59] & 1200 \\
\hline$\omega \pi \rho$ & $\omega$ exchange & $\frac{g_{\omega \pi \rho}^{2}}{4 \pi}=10.0$ & {$[18,60]$} & 1200 \\
\hline$N N a_{1}$ & $a_{1}$ exchange & $\sim f_{N N \pi}$ & & 1600 \\
\hline$a_{1} \pi \rho$ & $a_{1}$ exchange & $\sim g_{N N \rho}$ & & 1600 \\
\hline$N N \rho$ & $\rho$ exchange & $g_{N N \rho}, \kappa$ & & 1400 \\
\hline$\rho \rho \rho$ & $\rho$ exchange & $\sim g_{N N \rho}$ & & 1400 \\
\hline$N N \rho \rho$ & contact term & $\sim g_{N N \rho}^{2} \kappa$ & & 1200 \\
\hline$N \Delta \pi$ & $N$ exchange & $\frac{f_{N \Delta \pi}^{2}}{4 \pi}=0.36$ & {$[59]$} & 1600 \\
\hline$\Delta \Delta \pi$ & $\Delta$ exchange & $\frac{f_{\Delta \Delta \pi}^{2}}{4 \pi}=0.252$ & {$[61,62]$} & 1800 \\
\hline$N \Delta \rho$ & $\rho$ exchange & $\frac{f_{N \Delta \rho}^{2}}{4 \sqrt{2}}=20.45$ & {$[59]$} & 1400 \\
\hline$\Delta \Delta \rho$ & $\rho$ exchange & $\begin{array}{l}\frac{g_{\Delta \Delta \rho}}{4 \pi}=4.69 \\
\frac{g_{\Delta \Delta \rho}}{g^{2}}=6.1\end{array}$ & $\begin{array}{l}{[61,62]} \\
{[61,62]}\end{array}$ & 1400 \\
\hline$\pi \pi \rho$ & $\rho$ exchange & $\frac{g \rho \pi \pi}{4 \pi}=2.90$ & {$[63]$} & 1400 \\
\hline$N N \sigma$ & $N$ exchange & $\frac{g_{N N \sigma}^{2}}{4 \pi}=13$ & {$[37]$} & 1800 \\
\hline$\pi \pi \sigma$ & $\pi$ exchange & $\frac{g_{\pi \pi \sigma}^{2}}{4 \pi}=0.25$ & {$[64]$} & 1050 \\
\hline$N N \sigma$ & $\sigma$ exchange & $\sim g_{N N \sigma}$ & & 1700 \\
\hline$\sigma \sigma \sigma$ & $\sigma$ exchange & $\frac{g_{\sigma \sigma \sigma}^{2}}{4 \pi}=\mathbf{0 . 2 7 5}$ & & 1700 \\
\hline$N N \eta$ & $N$ exchange & $\frac{f_{N N \eta}^{2}}{4 \pi}=0.00934$ & [24] & 1500 \\
\hline$N N a_{0}$ & $a_{0}$ exchange & $\frac{g_{N N a_{0}} g_{\pi \eta a_{0}}}{4 \pi}=8.0$ & [24] & 1500 \\
\hline$\pi \eta a_{0}$ & $a_{0}$ exchange & & & 1500 \\
\hline
\end{tabular}

rily due to the presence of resonances in these partial waves, which are not yet included in the model. However, it is easy to see that the inclusion of the resonances in question alone will not help in the case of $P_{13}$ and $S_{31}$. This is because such resonance contributions will vanish again above the position of the resonance within the energy range given roughly by the width and the phase shift will change by $180^{\circ}$ (if the resonance contribution and the background have the same signs) or turn back to the background (if they have opposite signs). However, as one can see from Figs. 3 and 4, in the $P_{13}$ partial wave the phase goes in the opposite direction to the data, and in $S_{31}$ partial wave the deviation from the data at energies above the position of the resonance is huge.

The second problem of the $\pi N$ model of Krehl et al. is the presence of a long tail of the $S_{11}(1650)$ resonance. This leads to the undesirable feature that even at very low energies the $S_{11}$ phase shift is strongly influenced by this resonance, in conflict with chiral symmetry. As was shown by Weinberg 
TABLE IV. Parameters of the pole graphs: bare masses and coupling constants. The minus sign in parentheses indicates that the coupling constant is negative.

\begin{tabular}{lccccc}
\hline \hline & & \multicolumn{5}{c}{$f^{2} /(4 \pi)$} \\
Resonance & $\begin{array}{c}\text { Bare mass } \\
(\mathrm{MeV})\end{array}$ & $\pi N$ & $\pi \Delta$ & $\rho N$ & $\eta N$ \\
\hline$N_{S_{11}^{*}}^{*}(1535)$ & 2051 & 0.00045 & $1.09(-)$ & & 0.0247 \\
$N_{S_{11}}^{*}(1650)$ & 1919 & 0.0067 & & $0.046^{1}$ & \\
$N_{P_{13}}^{*}(1720)$ & 1910 & 0.0031 & $0.0085(-)$ & & $0.079(-)$ \\
$N_{D_{13}}^{*}(1520)$ & 2263 & 0.00037 & 0.0118 & 0.609 & 0.0008 \\
$\Delta_{(1232)}(123)$ & 1459 & 0.163 & & & \\
$\Delta_{S_{31}}(1620)$ & 2419 & 0.0154 & $2.91(-)$ & & \\
$\Delta_{P_{31}}(1910)$ & 2121 & 0.0043 & $0.007(-)$ & & \\
$\Delta_{D_{33}}(1700)$ & 2252 & 0.00038 & $0.03(-)$ & 0.011 & \\
\hline \hline
\end{tabular}

and Tomozawa [40,41] the isovector $s$-wave $\pi N$ scattering length is fully determined to leading order by the pion mass $m_{\pi}$ and pion decay constant $F_{\pi}$. Therefore, the presence of contributions related to the $N^{*}(1650)$ resonance at low energies is unnatural and physically hard to justify. Figure 5 shows the discussed effect. One can see that the low energy $S_{11}$ phase shift even changes its sign when all couplings to the $S_{11}(1650)$ resonance are switched off.

A detailed inspection of this problem revealed that the long tail of the $S_{11}(1650)$ resonance is predominantly due to the rather hard form factors used in the model of Krehl et al. and, in particular, in those diagrams contributing to the $\pi N$ $\rightarrow \rho N$ transition potential [there is a direct coupling of the $\rho N$ channel to the $\left.S_{11}(1650)\right]$. As was mentioned before, in the new model we want to avoid the use of extremely large cutoff masses anyway. A further reduction of the near threshold contribution from the $S_{11}(1650)$ resonance is achieved by choosing the derivative coupling for the $N_{S_{1}}^{*} N \pi$ vertex (see the Appendix) in analogy with the $N N \pi$ coupling. In the new model the missing strength at low energies is provided by the correlated $\pi \pi$ exchange in the $\sigma$ channel. It can be generated by allowing the subtraction constant, which occurs in the corresponding dispersion relations, cf. Eq. (31) in Ref. [22], and which was set to zero by hand in the old model [8], to assume a finite but still small value.

Now, let us consider the nonresonant part (or the background) of the new model. First one should note that the main contribution to the background at low energies is, of course, provided by diagrams that involve only the $\pi N$ channel. Therefore, we start by discussing the importance of various $\pi N$ graphs for the different partial waves. There are five diagrams in the $\pi N \rightarrow \pi N$ potential, cf. Figs. 1(a)-1(e): correlated $\pi \pi$ exchange in the $J=0, I=0(\sigma)$ and $J=1, I=1(\rho)$ channels, nucleon and $\Delta u$-channel exchanges, and the nucleon ( $s$-channel) pole diagram. It turned out that the contribution from the $\Delta$ exchange is very small in all partial waves. (As a consequence of this, we do not include $u$-channel graphs involving heavier resonances.) The $S$ waves are dominated by the $\rho$ and $\sigma$ exchanges. The nucleon exchange becomes important in higher partial waves. Note
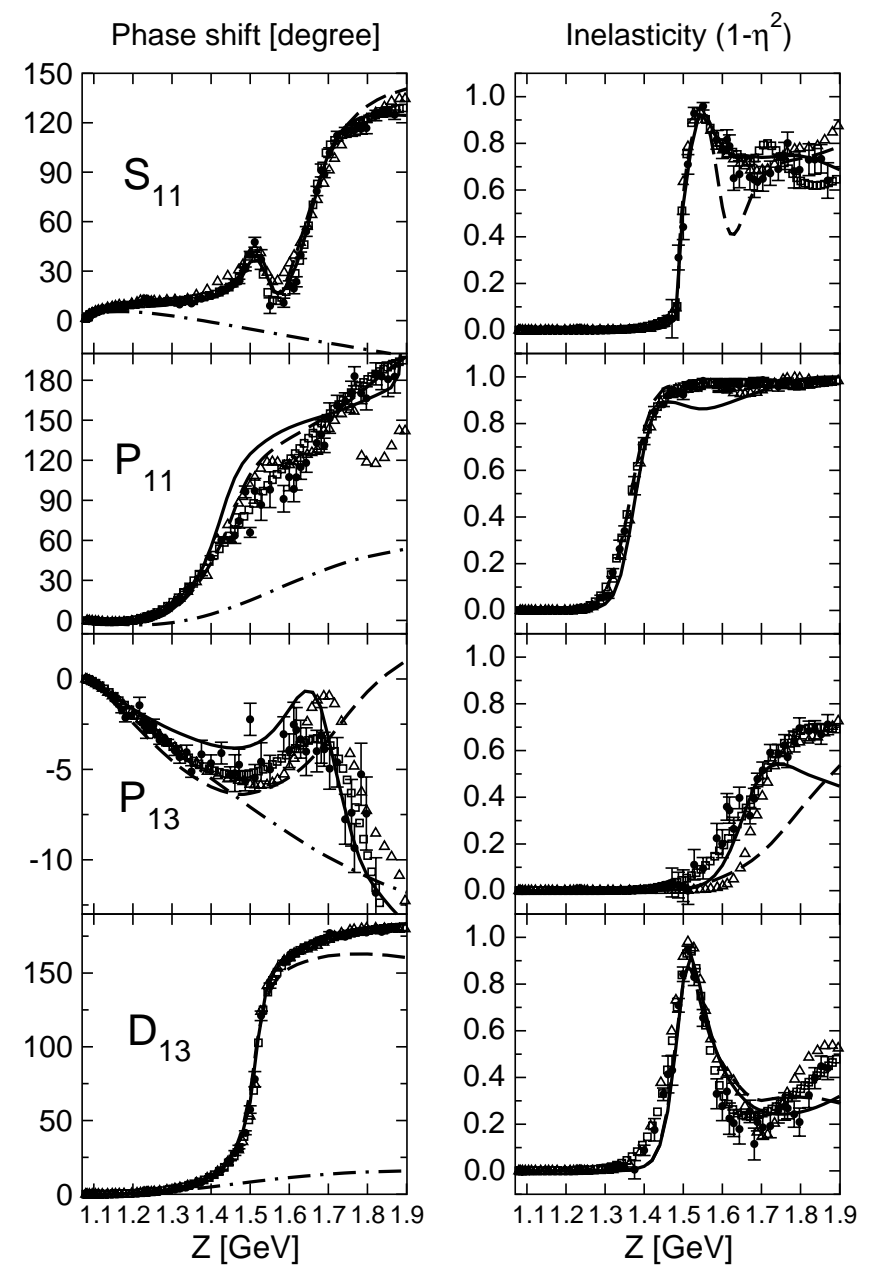

FIG. 3. The $\pi N$ phase shifts and inelasticities for the isospin $I$ $=1 / 2$ partial waves. The dashed curves show the results of the $\pi N$ model of Krehl et al. [8]. The dash-dotted curves represent the results based on the background contributions of our new model, as discussed in the text. The results of the full model are given by the solid lines. The data are from the phase-shift analyses KA84 [50], SM95 [2], and SE-SM95 [2].

also that the $\rho$ exchange alone provides such a strong attraction in the $P_{11}$ partial wave that it is almost sufficient for the formation of a resonance. However, it is partly canceled by the contribution from the nucleon pole. One should emphasize here that, in contrast to the old model, we do not have much freedom in varying the strength of the $\rho$ and $\sigma$ exchanges (except for the subtraction constant mentioned above), since their contributions at low energies are basically fixed due to our choice of the form factors (see Sec. II). Thus, the simultaneous description of the background in seven partial waves with a rather small number of parameters is to be considered as a success of our model (cf. dash-dotted lines in Figs. 3 and 4). We have not included the $P_{11}$ partial wave in these considerations, because there the coupling to the $\sigma N$ channel plays a rather important role.

As a confirmation for the quality of the background contribution we also looked at the phase shifts with $J=5 / 2$, cf. Fig. 6. These partial waves were not included in the fitting procedure and, therefore, are genuine predictions of our 

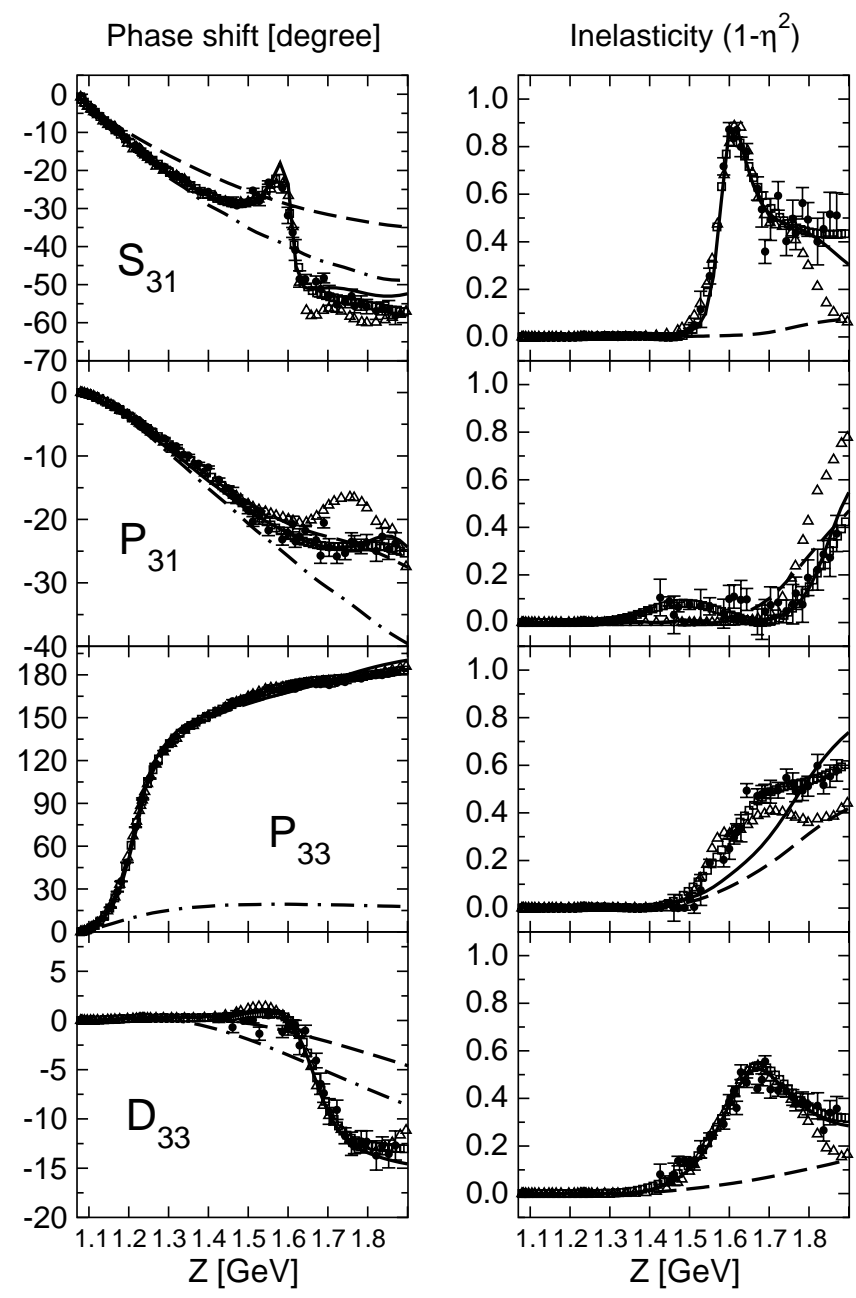

FIG. 4. The $\pi N$ phase shifts and inelasticities for the isospin $I$ $=3 / 2$ partial waves. Same description of curves and experiments as in Fig. 3.

model. It is evident that our results are quite in line with the general trend of the data (disregarding the resonance structures, of course).

The next step is the inclusion of the inelastic channels. The most important ones are those that represent effectively the $\pi \pi N$ channel, namely, $\rho N, \sigma N$, and $\pi \Delta$. The $\sigma N$ channel couples dominantly to the $P_{11} \pi N$ partial wave. It is a consequence of the parity difference between $\pi$ and $\sigma$, which implies that the $P_{11} \pi N$ partial wave couples to an $S$ wave in the $\sigma N$ system. In the course of adjusting the free parameters attraction is introduced into the $\sigma N$ channel and also a strong $\pi N \rightarrow \sigma N$ transition potential results. This, in turn, provides additional attraction in the $\pi N$ channel via coupled-channels effects and eventually leads to a dynamical generation of the $N^{*}(1440)$ (Roper) resonance in the $P_{11}$ partial wave. This mechanism and also its implications for the Roper resonance were discussed extensively in previous studies $[8,24]$ by the Jülich group and, therefore, we do not repeat the arguments here. However, it is certainly reassuring that also within the new model the Roper resonance turns out to be dynamically generated, and no genuine $N^{*}(1440)$ (three quark) resonance is needed to explain the $P_{11}$ partial wave.
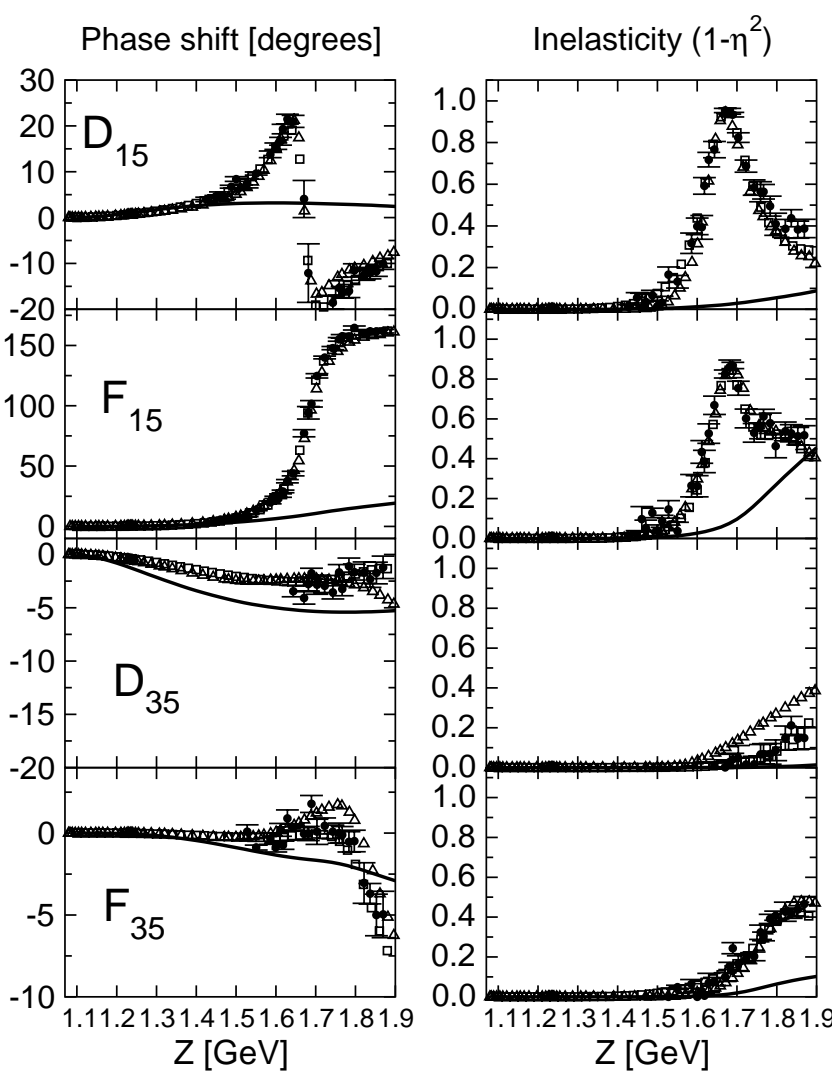

FIG. 5. Phase shifts and inelasticities for $\pi N$ partial waves with $J=5 / 2$. Same description of curves and experiments as in Fig. 3.

The channels $\rho N$ and $\pi \Delta$ are important for the inelasticities at high energies in all partial waves, but, in particular, in the $D_{13}, P_{31}$, and $P_{33}$. In the $P_{33}$ partial wave there are no resonances in this energy region that couple strongly to the $\pi N$ system [29]. Thus, coupling to these channels via $t$ - and $u$-channel exchange diagrams is the only source of inelasticity in the $P_{33} \pi N$ partial wave. The most important diagrams for the $P_{33}$ inelasticity are the $\rho$ exchange in the $\pi N \rightarrow \pi \Delta$ potential and, partly, the nucleon exchange in the $\pi N \rightarrow \rho N$ potential. One should mention here also the $\pi$-exchange diagram in the $\pi N \rightarrow \rho N$ transition. It turns out to be much too strong in the $P_{13}$ and $S_{11}$ partial waves, independent of the cutoff used. Its contribution alone produces a very strong cusp in the region of the $\rho N$ threshold in the $S_{11}$ phase shift, and drastically modifies the behavior of the $P_{13}$ phase shift, bending it upwards. Luckily the $\pi$-exchange contribution is canceled to a large extent by the $\pi N \rightarrow \rho N$ contact term from the Wess-Zumino Lagrangian, and also by the $\omega$-exchange diagram. Ultimately, on the whole, the phase shifts are not too much affected by the inelastic channels.

The final step in the description of the elastic $\pi N$ data consists in adding the resonance terms. We included resonances in all partial waves except for the $P_{11}$ where our model reproduces the phase shift and inelasticity, including the structure associated with the Roper resonance, dynamically via a strong coupling to the $\sigma N$ channel, as already mentioned above. In the $S_{11}$ partial wave there are two resonances, namely, the $N^{*}(1535)$ and the $N^{*}(1650)$. The former dominates the near threshold $\pi N \rightarrow \eta N$ cross section. (The 

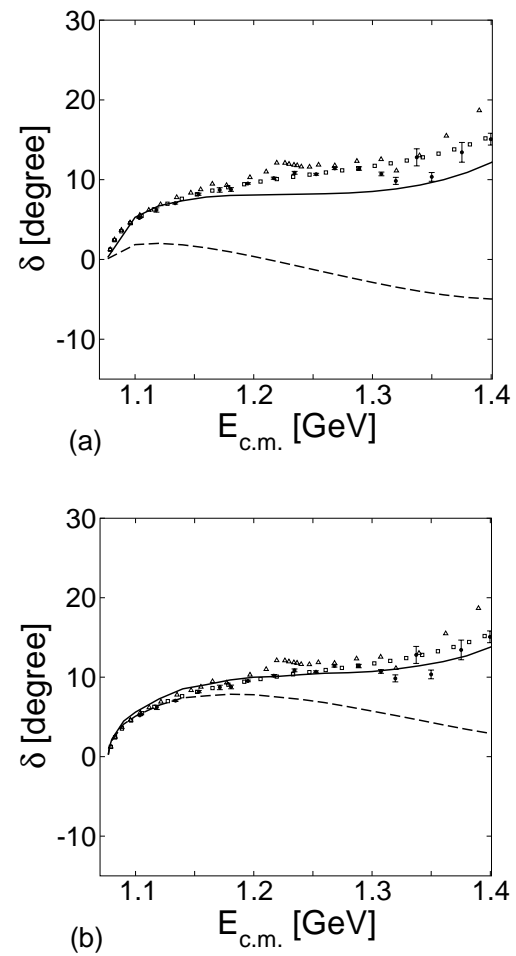

FIG. 6. $\pi N$ phase shift in the $S_{11}$ partial wave. The results of the model of Krehl et al. [8] are shown at the top and those of the new model at the bottom. The curves correspond to the full model (solid line) and to the full model with the contribution of the $S_{11}(1650)$ resonance switched off (dashed line). The data are from the phaseshift analyses KA84 [50], SM95 [2], and SE-SM95 [2].

$\eta N$ channel will be discussed in detail in the following section.) As can be seen from the parameters given in Table IV, among the effective $\pi \pi N$ channels, the $\pi \Delta$ channel is allowed to couple to most of the resonances. This channel becomes relevant already at rather low energies (in contrast to the $\rho N$ channel) and it can contribute to both $(I=1 / 2$ and $I=3 / 2$ ) isospin states. Since we cannot calculate $\pi N \rightarrow \pi \pi N$ observables directly at the moment (due to technical difficulties that arise from three-body singularities)—which would allow us to further constrain the relative importance of the different $\pi \pi N$ channels-we choose this particular channel for describing the bulk of the $\pi \pi N$ part of the $\pi N$ inelasticity. However, in addition, the $\rho N$ channel needs to be coupled to some resonances, namely, $D_{13}(1520), S_{11}(1650)$, and $D_{33}(1700)$. In these cases the different energy behavior resulting from the $\rho N$ channel is required for a satisfactory description of the experimental phase shifts as well as the inelasticities.

The position of the $P_{31}(1910)$ resonance is located already above the energy region we are interested in (which is from $\pi N$ threshold up to $\sim 1.9 \mathrm{GeV}$ ). Nevertheless it was included because its tail still influences noticeably the energy region around $1.8 \sim 1.9 \mathrm{GeV}$.

Note that, among others, the inelasticity in the $P_{13}$ partial wave shows an incorrect trend at higher energies, and the data are underestimated. A similar, but less pronounced deficiency can be found in the $D_{13}$ inelasticity. Some authors claim, that there is a sizable contribution from the $\omega N$ chan-
TABLE V. The $s$ - and $p$-wave $\pi N$ scattering lengths and volumes in terms of $m_{\pi^{+}}^{-(2 L+1)}$.

\begin{tabular}{cccc}
\hline \hline & This work & Reference [65] & SM95 [2] \\
\hline$S_{11}$ & 0.195 & $0.173 \pm 0.003$ & 0.175 \\
$S_{31}$ & -0.110 & $-0.101 \pm 0.004$ & -0.087 \\
$P_{11}$ & -0.089 & $-0.081 \pm 0.002$ & -0.068 \\
$P_{31}$ & -0.046 & $-0.045 \pm 0.002$ & -0.039 \\
$P_{13}$ & -0.031 & $-0.030 \pm 0.002$ & -0.022 \\
$P_{33}$ & 0.209 & $0.214 \pm 0.002$ & 0.209 \\
\hline \hline
\end{tabular}

nel, which opens at around $1.7 \mathrm{GeV}$, to these particular partial waves [42]. Therefore, the inclusion of the $\omega N$ channel might improve the description of these data.

Finally, let us mention that also the low energy parameters of $\pi N$ scattering are in reasonable agreement with available data, as it should be, since we fit our model to the phase-shift analyses. The $S$ and $P$-wave scattering lengths and volumes are collected in Table V.

\section{Description of the $\eta N$ channel}

The reaction $\pi N \rightarrow \eta N$ near the $\eta N$ threshold is closely related to the properties of the $N^{*}(1535)$ resonance. The total cross section of this reaction has a very pronounced peak structure at the position of the resonance (cf. Fig. 7). In the previous version of the Jülich $\pi N$ model the total $\pi^{-} p \rightarrow \eta n$ cross section was overestimated by about $20-30 \%$ around the maximum. The reason for this deficiency is that only the $\pi N$ and $\eta N$ channels were allowed to couple to the $N^{*}(1535)$ resonance. Therefore, in order to describe the $S_{11} \pi N$ amplitude one had to generate basically the whole inelasticity in this partial wave by the coupling to the $\eta N$ channel. Indeed, the contribution of the $S_{11}$ partial wave to the inelastic $\pi^{-} p$ cross section is given by

$$
\sigma_{i n}=\frac{2 \pi}{3 k_{1}^{2}}\left(1-\eta^{2}\right),
$$

which amounts to $\sigma_{\text {in }} \sim 3.5 \mathrm{mb}$ at the maximum using the inelasticity $\eta$ as given by the phase-shift analysis. How-

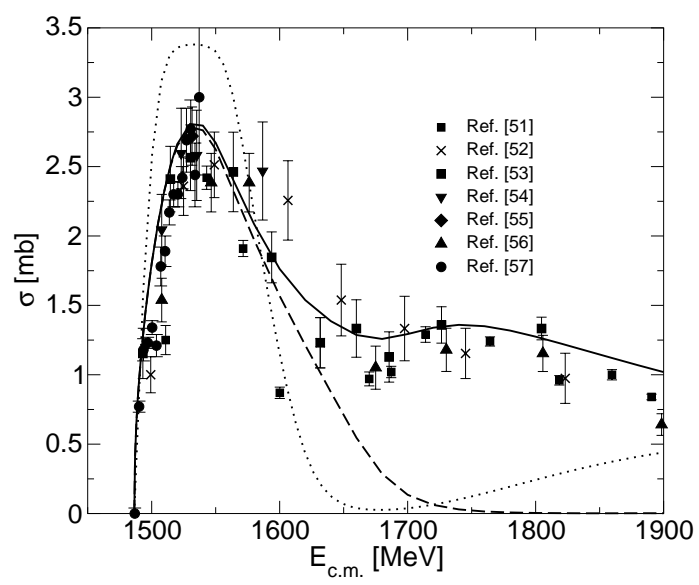

FIG. 7. $\pi^{-} p \rightarrow \eta n$ total cross section. The solid line corresponds to the full calculation. The dashed line indicates the pure $s$-wave contribution. The results of the old model are shown as a dotted line (only $s$ wave). The data are from Refs. [51-57]. 
ever, the experimental $\pi^{-} p \rightarrow \eta n$ cross section is always below $3 \mathrm{mb}$, cf. Fig. 7. Thus, it is clear that there must be contributions of other channels to the $S_{11}$ inelasticity. The only other channel which is open at energies around the $\eta$ threshold is the $\pi \pi N$ channel. Indeed, the $\pi \pi N$ channel was found to be important in an analysis of the $\pi N$ $\mathrm{S}$-waves within the chiral unitary approach of Inoue et al. [43]. Accordingly, we introduce a coupling of the $\pi \Delta$ system-which in our model is one of the effective channels that represent the $\pi \pi N$ channel-to the $N^{*}(1535)$ resonance. This enables us to describe simultaneously the total $\pi^{-} p$ $\rightarrow \eta n$ cross section and the inelasticity in the $S_{11}$ partial wave in the resonance region, as can be seen in Figs. 7 and 3, respectively.

The inclusion of an $N^{*} \Delta \pi$ coupling improves also the description of the $S_{11}$ inelasticity above the position of the $N^{*}(1535)$ resonance. In Fig. 3 one can see that the old Jülich model produces a strong dip in the $S_{11}$ inelasticity, which then leads to a similar dip in the $S$-wave $\pi^{-} p \rightarrow \eta n$ cross section. We found that the origin of this behavior is essentially a unitarity constraint from the $\pi N$ channel. It can be easily understood schematically if we assume a two-channel problem involving only the $\pi N$ and $\eta N$ systems. We also assume that, apart from the $N^{*}(1535)$ resonance (whose contribution drops quickly when one moves away from its peak), there is some background contribution to the $\pi N$ $\rightarrow \eta N$ transition potential and that at the same time (which is the crucial point) the direct $\eta N \rightarrow \eta N$ potential is negligibly small. (These conditions are satisfied in the old Jülich model.) Then the $\pi N \rightarrow \eta N T$ matrix (we consider only the $S_{11}$ partial wave) is given by

$$
T_{\pi N \rightarrow \eta N}=V_{\pi N \rightarrow \eta N}\left(1+G_{0} T_{\pi N \rightarrow \pi N}\right),
$$

which can be reexpressed in the form (see, e.g., Ref. [44])

$$
T_{\pi N \rightarrow \eta N}=\left(1+\left(\beta+i k_{\pi N}\right) \frac{\eta e^{2 i \delta}-1}{2 i k_{\pi N}}\right) V_{\pi N \rightarrow \eta N} .
$$

Here $\beta$ is the inverse of the characteristic range of interaction, which is determined by the principal value integral, $\delta$ and $\eta$ are the $S_{11}$ phase shift and inelasticity parameter, and $k_{\pi N}$ is the on-shell momentum in the $\pi N$ channel. Let us now examine under what circumstances we can have $T_{\pi N \rightarrow \eta N}=0$. Given our simplifying model assumption, the condition $T_{\pi N \rightarrow \eta N}=0$ implies that $\eta=1$ and consequently $\beta$ is purely real. Then, it is convenient to rewrite Eq. (12) as

$$
T_{\pi N \rightarrow \eta N}=e^{i \delta} \sqrt{1+\beta^{2} / k_{\pi N}^{2}} \sin (\gamma-\alpha) V_{\pi N \rightarrow \eta N},
$$

where $\gamma=\arctan \left(\beta / k_{\pi N}\right)$ and $\alpha=\delta-\pi / 2$. Note that in the specific situation we discuss that the phase $\delta$ crosses $\pi / 2(\alpha=0)$, due to the presence of the $N^{*}(1650)$ resonance in the $\pi N \rightarrow \pi N$ interaction, and then continues to rise rapidly, whereas $\beta$ is a smooth function of $k_{\pi N}$ and has a typical value in the order of several hundred $\mathrm{MeV}$ (the exact value is, of course, model dependent), so that in the region of interest we have $\gamma \lesssim 1$. It is thus easy to convince oneself that the expression in Eq. (13) equals zero at some energy above (but not far from) the position of the
$N^{*}$ (1650) resonance. Expanding $T_{\pi N \rightarrow \eta N}$ in powers of $Z$ $-Z_{0}$, where $Z_{0}$ is the position of the "zero," one can see that the $\pi^{-} p \rightarrow \eta n$ cross section is proportional to $(Z$ $\left.-Z_{0}\right)^{2}$-which explains the structure of the dip in the cross section exhibited by the old Jülich model (dotted line in Fig. 7). It is interesting to note that the same effect can be found in other model analyses, e.g., in the ones by Gridnev and Kozlenko [26] and by the Giessen group [27,28] In general, when there are more than two channels, $\beta$ becomes complex and the cross section at the dip will be finite-but it will still be small (provided that the inelasticity is not too large).

In our model the $S_{11}$ inelasticity in the energy region around the $\eta$ threshold is partly determined by the $N^{*}(1535) \Delta \pi$ coupling. In the $\Delta \pi$ system this resonance couples to a pure $D$ wave. Because of this the maximum of the $\pi N \rightarrow \pi \Delta$ transition cross section is shifted to somewhat higher energies as compared to the resonance energy. Its contribution to the inelasticity is likewise shifted to somewhat higher energies and fills up the dip that can be seen in the $S_{11}$ inelasticity predicted by the old model, cf. Fig. 3. It also smoothens out the effect discussed above and, therefore, we can achieve a fairly realistic description of the energy dependence of $\sigma_{\pi^{-} p \rightarrow \eta n}$ over the region of the $N^{*}(1650)$ resonance. Specifically, we do not get this strong double hump structure prominently visible in the model analysis of Ref. [27], cf. their Fig. 7.

Let us now look at the energy dependence of the total cross section over a wider energy range and also at the $\pi^{-} p \rightarrow \eta n$ differential cross section in order to examine the importance of higher partial waves. To include the effect of higher partial waves we introduced a coupling of the $\eta N$ system to the $P_{13}(1720)$ and $D_{13}(1520)$ resonances. These are the most pronounced resonances in the energy region below $1.9 \mathrm{GeV}$ that couple strongly to the $\pi N$ system. Note that there are other three-star $N^{*}$ resonances [29] in this region. However, we do not include these because their coupling to the $\pi N$ channel is very weak and therefore their parameters cannot be sufficiently constrained from the $\pi N$ data.

At energies below $1.6 \mathrm{GeV}$ the slight deviation of the differential cross section from the isotropic distribution can be easily described by the interference of the $D_{13}$ resonance with the $S$-wave amplitude [45], cf. Fig. 8. For the total cross section the $D_{13}$ contribution is of minor importance. Above $1.6 \mathrm{GeV}$ the total cross section can be described by introducing a coupling of the $\eta N$ system to the $P_{13}(1720)$ resonance, as is evidenced by the results shown in Fig. 7. However, as is obvious from Fig. 9, this coupling alone is not sufficient to achieve also good agreement with the data for the differential cross section in this energy region. Most likely this points to missing contributions from higher partial waves, and specifically from $J=5 / 2$ resonances. At present we do not aim to include these. We would also like to remark that the existing data do not allow one to discriminate between different partial-wave contributions-one would need to know polarization observables for this purpose.

Finally, we want to draw attention to the fact that in our model there is also a background contribution to the $\pi N$ $\rightarrow \eta N$ transition interaction which is provided by $t$-channel 

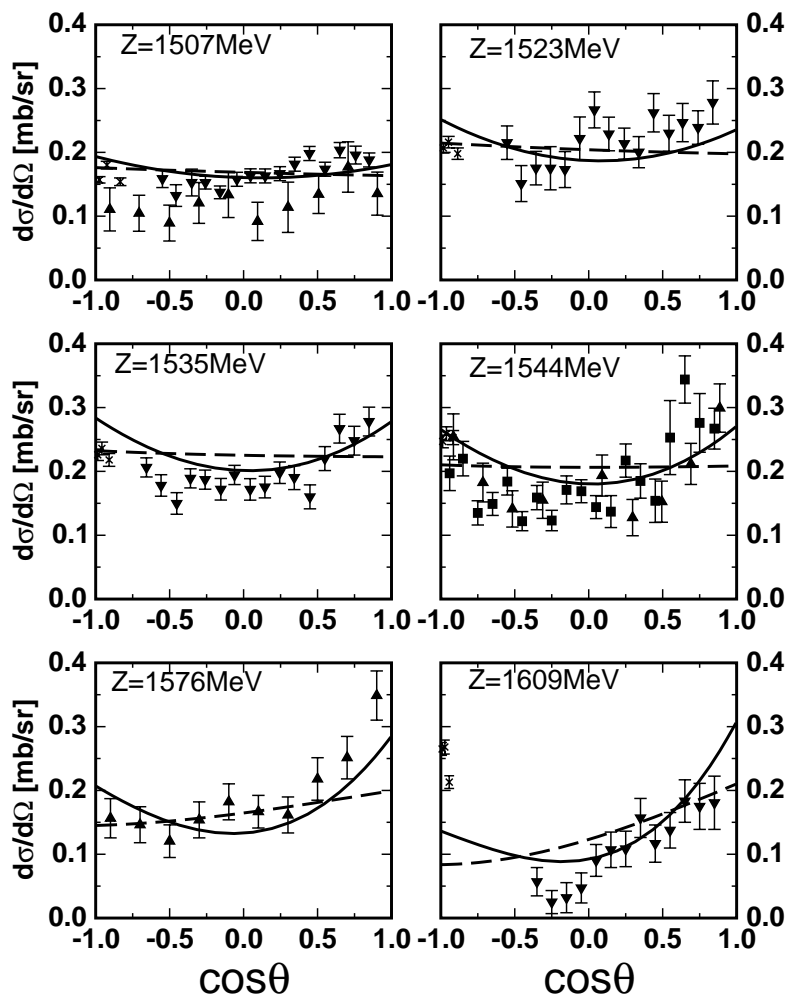

FIG. 8. $\pi^{-} N \rightarrow \eta n$ differential cross section at energies close to the $\eta N$ threshold. The solid curve corresponds to the full result. The dashed line corresponds to the case when the $D_{13}$ partial wave is switched off. The data are from Refs. [51] ( $\square)$, [54] ( $)$, [56] ( $\mathbf{\Delta})$, and $[58](\times)$.

exchange of the $a_{0}(980)$ meson, cf. Fig. 2(b). However, the role of $a_{0}$ exchange is now strongly reduced as compared to the old Jülich model, mainly because in the present model we avoid large values of the cutoff mass. In any case, the influence of the $a_{0}(980)$ meson is suppressed at energies above the $N^{*}(1535)$ resonance due to the mechanism discussed above.

We also want to present the $\eta N$ effective range parameters predicted by our model. They are

$$
\begin{aligned}
& a_{\eta N}=(0.41+i 0.26) \mathrm{fm}, \\
& r_{\eta N}=(-3.4+i 0.4) \mathrm{fm} .
\end{aligned}
$$

Obviously, our result for $\operatorname{Re}\left(a_{\eta N}\right)$ is at the lower end of the spectrum of values that one can find in the literature, cf., e.g., the compilation given in Table I of Ref. [46]. In fact, it is even slightly lower than the one of the old Jülich model, which yields $a_{\eta N}=(0.42+i 0.34) \mathrm{fm}$. However, we want to emphasize that such a value is pretty much in line with conclusions drawn from recent analyses of the $\eta N$ final state interaction in the reactions $\gamma d \rightarrow n p \eta[46]$ and $p n \rightarrow d \eta[47,48]$.

\section{SUMMARY}

We have presented results of an extended and improved version of the Jülich $\pi N$ model. The model is based on the
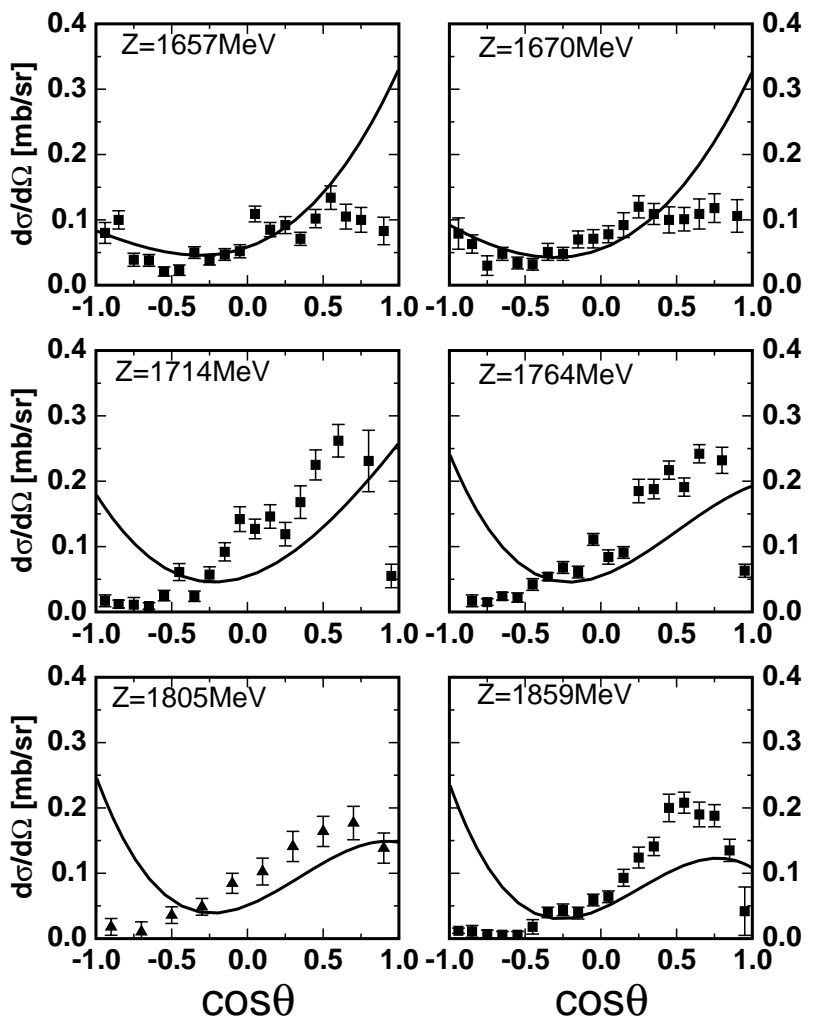

FIG. 9. $\pi^{-} N \rightarrow \eta n$ differential cross section at higher energies. The data are from Refs. [51] $(\square)$, [54] $(\boldsymbol{\nabla}),[56](\mathbf{\Delta})$, and [58]( $\times)$.

meson-exchange picture and it is derived in its main part from the phenomenological Wess-Zumino Lagrangian, consistent with chiral symmetry. The $\pi N$ interaction in the scalar-isoscalar and vector-isovector channels is calculated by means of dispersion relations from correlated $\pi \pi$ exchange in order to constrain the contributions of the corresponding $\sigma$ and $\rho$ exchanges. In the present work ambiguities in the treatment of dispersion relations (cf. Sec. III B of Ref. [22]) are even further reduced by a choice of the form factors which does not modify the strength and $t$ dependence of the interaction at low energies. In addition, some more improvements have been implemented. In particular, we now use derivative coupling for the $S_{11} N^{*}$ resonances at the $\pi N$ and $\eta N$ vertices, as is demanded by chiral symmetry anyway. We also include some more resonance diagrams, specifically for the $S_{13}(1620), \quad P_{13}(1720), \quad P_{13}(1910), \quad D_{13}(1520)$, and $D_{33}(1700)$ resonances.

The potential constructed in this way was unitarized in a coupled channels-Lippmann-Schwinger equation to obtain the reaction amplitudes for various processes. The reaction channels included in the present investigation are $\pi N, \eta N$, $\sigma N, \rho N$, and $\pi \Delta$, where the latter three channels are understood as an effective description of the physical $\pi \pi N$ state.

With the new model an excellent quantitative reproduction of the $\pi N$ phase shifts and inelasticity parameters in the energy region up to $1.9 \mathrm{GeV}$ and for total angular momenta $J \leqslant 3 / 2$ was achieved. In addition, a good description of the background in the $J=5 / 2$ partial waves was obtained automatically. As far as the $P_{11}$ partial wave is concerned we confirm the results of our earlier investigations that the struc- 
ture associated with the Roper resonance is generated dynamically by the model so that no genuine $N^{*}(1440)$ (threequark) resonance diagram needs to be included.

As the main new aspect we studied in detail the coupling of the $\pi N$ system to the $\eta N$ channel. First of all we showed that the overestimation of the $\pi^{-} p \rightarrow \eta n$ cross section in the region of the $N^{*}(1535)$ resonance by the old Jülich model can be removed by introducing additional flux from the $\pi N$ to the $\pi \Delta$ channel. Furthermore, the inclusion of the $\eta N$ coupling to the $P_{13}(1720)$ resonance turned out to lead to a significant improvement of the total cross section at higher energies. At the same time the puzzle of the dip in the $S_{11}$ inelasticity, present in the old Jülich model but also in other models in the literature [26,27], could be explained. The origin of this deficiency turned out to be an almost model independent effect of coupled-channels unitarity constraints. We also improved the description of the $\pi^{-} p \rightarrow \eta n$ differential cross section. A remaining discrepancy with the data at higher energies is most likely caused by contributions from partial waves with $J>3 / 2$ which are not included in our model calculation. Note that a detailed partial-wave analysis of this reaction at such energies is presently impossible because of the lack of data on polarization observables.

The model in its present form enables a straightforward inclusion of further reaction channels, and specifically those nearest in energy, namely, $K \Lambda, K \Sigma$, and $\omega N$. Such an extension of the present model in this direction is planned for the future.

\section{ACKNOWLEDGMENTS}

We thank S. Krewald and I. R. Afnan for stimulating discussions.

\section{APPENDIX: THE PSEUDOPOTENTIAL}

In this appendix we give the expressions for those contributions to the interaction potentials that differ from our earlier work [8]. For convenience we also summarize here all pole diagrams since most of them were not included in the old model. All other expressions for the pseudopotential can be found in Appendix A of Ref. [8]. The notation for the different particles and their momenta is given in Fig. 1. $E_{1}$, $E_{3}, \omega_{2}$, and $\omega_{4}$ indicate on-mass-shell energies of baryons 1 and 3 and those of mesons 2 und 4, respectively:

$$
E_{i}=\sqrt{m_{i}^{2}+p_{i}^{2}}, \omega_{i}=\sqrt{m_{i}^{2}+p_{i}^{2}} .
$$

$q$ is the four-momentum of the intermediate particle. The tensor operator $P^{\mu \nu}$ is given in Eq. (A12) of Ref. [8].

Since we work in time-ordered perturbation theory, all the potentials contain the normalization factor

$$
\kappa=\frac{1}{(2 \pi)^{3}} \sqrt{\frac{m_{1}}{E_{1}} \frac{m_{3}}{E_{3}}} \sqrt{\frac{1}{2 \omega_{2} 2 \omega_{4}}} .
$$

$$
\text { 1. } \pi N \rightarrow \pi N
$$

Correlated $\pi \pi$ exchange in the $\sigma$ channel [Fig. 1(c)]:

$$
-\kappa \bar{u}\left(\vec{p}_{3}, \lambda_{3}\right) u\left(\vec{p}_{1}, \lambda_{1}\right)\left[A_{0}+16\left(-2 p_{2 \mu} p_{4}^{\mu}\right) \int d t^{\prime} \frac{\operatorname{Im}\left[f_{+}^{0}\left(t^{\prime}\right)\right]}{\left(t^{\prime}-2 m_{\pi}^{2}\right)\left(t^{\prime}-4 m_{N}^{2}\right)} P\left(t^{\prime}\right)\right] I F_{\sigma t}(I),
$$

where $P\left(t^{\prime}\right)=\left(1 / 2 \omega_{t^{\prime}}\right)\left(\left[1 /\left(E-\omega_{2}-E_{3}-\omega_{t^{\prime}}\right)\right]+\left[1 /\left(E-\omega_{4}-E_{1}-\omega_{t^{\prime}}\right)\right]\right), \omega_{t^{\prime}}=\sqrt{q^{2}+t^{\prime}}$, and $f$ is a Frazer-Fulco amplitude $[25,49]$. The isospin coefficients are equal to $I F_{\sigma t}(1 / 2)=1$ and $I F_{\sigma t}(3 / 2)=1$.

$N^{*}\left(S_{11}, S_{31}\right)$ pole diagrams [Fig. $\left.1(\mathrm{~g})\right]$ :

$$
\kappa \frac{f_{N^{*} N \pi}^{2}}{m_{\pi}^{2}} \bar{u}\left(\vec{p}_{3}, \lambda_{3}\right) p_{4} \frac{1}{2 m_{N^{*}}^{0}} \frac{q+m_{N^{*}}^{0}}{E-m_{N^{*}}^{0}} p_{2} u\left(\vec{p}_{1}, \lambda_{1}\right) I F_{N^{*} s}(I)
$$

Nucleon, $N^{*}\left(P_{31}\right)$ pole diagrams:

$$
\kappa \frac{f_{N^{*} N \pi}^{2}}{m_{\pi}^{2}} \bar{u}\left(\vec{p}_{3}, \lambda_{3}\right) \gamma^{5} p_{4} \frac{1}{2 m_{N^{*}}^{0}} \frac{q+m_{N^{*}}^{0}}{E-m_{N^{*}}^{0}} \gamma^{5} p_{2} u\left(\vec{p}_{1}, \lambda_{1}\right) I F_{N^{*} s}(I) .
$$

$N^{*}\left(P_{13}, P_{33}\right)$ pole diagrams:

$$
\kappa \frac{f_{N^{*} N \pi}^{2}}{m_{\pi}^{2}} \bar{u}\left(\vec{p}_{3}, \lambda_{3}\right) p_{4 \mu} \frac{1}{2 m_{N^{*}}^{0}} \frac{P^{\mu \nu}(q)}{E-m_{N^{*}}^{0}} p_{2 \nu} u\left(\vec{p}_{1}, \lambda_{1}\right) I F_{N^{*} s}(I) .
$$

$N^{*}\left(D_{13}, D_{33}\right)$ pole diagrams:

$$
\kappa \frac{f_{N^{*} N \pi}^{2}}{m_{\pi}^{4}} \bar{u}\left(\vec{p}_{3}, \lambda_{3}\right) \gamma^{5} p_{4} p_{4 \mu} \frac{1}{2 m_{N^{*}}^{0}} \frac{P^{\mu \nu}(q)}{E-m_{N^{*}}^{0}} \gamma^{5} p_{2} p_{2 \nu} u\left(\vec{p}_{1}, \lambda_{1}\right) I F_{N^{*} s}(I),
$$

$I F_{N^{*}{ }^{*}}(1 / 2)=3, I F_{N^{*}{ }^{*}}(3 / 2)=1$. 


\section{2. $\pi N \rightarrow \eta N$}

$N^{*}\left(S_{11}\right)$ pole diagram:

$$
\kappa \frac{f_{N^{*} N \pi} f_{N^{*} N \eta}}{m_{\pi}^{2}} \bar{u}\left(\vec{p}_{3}, \lambda_{3}\right) p_{4} \frac{1}{2 m_{N^{*}}^{0}} \frac{\phi+m_{N^{*}}^{0}}{E-m_{N^{*}}^{0}} p_{2} u\left(\vec{p}_{1}, \lambda_{1}\right) I F_{N^{*} s}(I) .
$$

$N^{*}\left(P_{13}\right)$ pole diagram:

$$
\kappa \frac{f_{N^{*} N \pi} f_{N^{*} N \eta}}{m_{\pi}^{2}} \bar{u}\left(\vec{p}_{3}, \lambda_{3}\right) p_{4 \mu} \frac{1}{2 m_{N^{*}}^{0}} \frac{P^{\mu \nu}(q)}{E-m_{N^{*}}^{0}} p_{2 \nu} u\left(\vec{p}_{1}, \lambda_{1}\right) I F_{N^{*}{ }^{*}}(I) .
$$

$N^{*}\left(D_{13}\right)$ pole diagram:

$$
\kappa \frac{f_{N^{*} N \pi} f_{N^{*} N \eta}}{m_{\pi}^{4}} \bar{u}\left(\vec{p}_{3}, \lambda_{3}\right) \gamma^{5} p_{4} p_{4 \mu} \frac{1}{2 m_{N^{*}}^{0}} \frac{P^{\mu \nu}(q)}{E-m_{N^{*}}^{0}} \gamma^{5} p_{2} p_{2 \nu} u\left(\vec{p}_{1}, \lambda_{1}\right) I F_{N^{*} s}(I) .
$$

$I F_{N^{*}}(1 / 2)=\sqrt{3}$.

$$
\text { 3. } \pi N \rightarrow \rho N
$$

$N^{*}\left(S_{11}\right)$ pole diagram:

$$
-i \kappa \frac{f_{N^{*} N \pi} g_{N^{*} N \rho}}{m_{\pi}} \bar{u}\left(\vec{p}_{3}, \lambda_{3}\right) \gamma^{5} \xi^{*}\left(\vec{p}_{4}, \lambda_{4}\right) \frac{1}{2 m_{N^{*}}^{0}} \frac{q+m_{N^{*}}^{0}}{E-m_{N^{*}}^{0}} p_{2} u\left(\vec{p}_{1}, \lambda_{1}\right) I F_{N^{*} s}(I) .
$$

$N^{*}\left(D_{13}, D_{33}\right)$ pole diagrams

$$
: i \kappa \frac{f_{N^{*} N \pi} f_{N^{*} N \rho}}{m_{\pi}^{2} m_{\rho}} \bar{u}\left(\vec{p}_{3}, \lambda_{3}\right)\left[p_{4} \epsilon_{\mu}^{*}\left(\vec{p}_{4}, \lambda_{4}\right)-p_{4 \mu} k^{*}\left(\vec{p}_{4}, \lambda_{4}\right)\right] \frac{P^{\mu \nu}(q)}{2 m_{N^{*}}\left(E-m_{N^{*}}^{0}\right)} p_{2} \gamma_{\nu}^{5} p_{2} u\left(\vec{p}_{1}, \lambda_{1}\right) I F_{N^{*}{ }^{*}}(I)
$$

$I F_{N^{*}{ }^{*}}(1 / 2)=3$ and $I F_{N^{*}{ }^{*}}(3 / 2)=1$.

$$
\text { 4. } \pi N \rightarrow \pi \Delta
$$

$N^{*}\left(S_{11}, S_{31}\right)$ pole diagrams:

$$
\kappa \frac{f_{N^{*} N \pi} f_{N^{*} \Delta \pi}}{m_{\pi}^{2}} \bar{u}_{\mu}\left(\vec{p}_{3}, \lambda_{3}\right) p_{4}^{\mu} \gamma^{5} \frac{1}{2 m_{N^{*}}^{0}} \frac{q+m_{N^{*}}^{0}}{E-m_{N^{*}}^{0}} p_{2} u\left(\vec{p}_{1}, \lambda_{1}\right) I F_{N^{*} s}(I)
$$

$N^{*}\left(P_{31}\right)$ pole diagram:

$$
\kappa \frac{f_{N^{*} N \pi} f_{N^{*} \Delta \pi}}{m_{\pi}^{2}} \bar{u}_{\mu}\left(\vec{p}_{3}, \lambda_{3}\right) p_{4}^{\mu} \frac{1}{2 m_{N^{*}}^{0}} \frac{q+m_{N^{*}}^{0}}{E-m_{N^{*}}^{0}} \gamma^{5} p_{2} u\left(\vec{p}_{1}, \lambda_{1}\right) I F_{N^{*} s}(I)
$$

$N^{*}\left(P_{13}\right)$ pole diagram:

$$
\kappa \frac{f_{N^{*} N \pi} f_{N^{*} \Delta \pi}}{m_{\pi}^{2}} \bar{u}_{\mu}\left(\vec{p}_{3}, \lambda_{3}\right) \gamma^{5} p_{4} \frac{1}{2 m_{N^{*}}^{0}} \frac{P^{\mu \nu}(q)}{E-m_{N^{*}}^{0}} p_{2 \nu} u\left(\vec{p}_{1}, \lambda_{1}\right) I F_{N^{*}{ }^{*}}(I)
$$

$N^{*}\left(D_{13}, D_{33}\right)$ pole diagrams:

$$
-\kappa \frac{f_{N^{*} N \pi} f_{N^{*}} \Delta \pi}{m_{\pi}^{3}} \bar{u}_{\mu}\left(\vec{p}_{3}, \lambda_{3}\right) p_{4} \frac{1}{2 m_{N^{*}}^{0}} \frac{P^{\mu \nu}(q)}{E-m_{N^{*}}^{0}} \gamma^{5} p_{2} p_{2 \nu} u\left(\vec{p}_{1}, \lambda_{1}\right) I F_{N^{*} s}(I) .
$$

$I F_{N^{*}{ }^{*}}(1 / 2)=-\sqrt{6}$ and $I F_{N^{*}{ }_{s}}(3 / 2)=\sqrt{\frac{5}{3}}$.

$$
\text { 5. } \eta N \rightarrow \eta N
$$

$N^{*}\left(S_{11}\right)$ pole diagram: 


$$
\kappa \frac{f_{N^{*} N \eta}^{2}}{m_{\pi}^{2}} \bar{u}\left(\vec{p}_{3}, \lambda_{3}\right) p_{4} \frac{1}{2 m_{N^{*}}^{0}} \frac{q+m_{N^{*}}^{0}}{E-m_{N^{*}}^{0}} p_{2} u\left(\vec{p}_{1}, \lambda_{1}\right) I F_{N^{*} s}(I)
$$

$N^{*}\left(P_{13}\right)$ pole diagram:

$$
\kappa \frac{f_{N^{*} N \eta}^{2}}{m_{\pi}^{2}} \bar{u}\left(\vec{p}_{3}, \lambda_{3}\right) p_{4 \mu} \frac{1}{2 m_{N^{*}}^{0}} \frac{P^{\mu \nu}(q)}{E-m_{N^{*}}^{0}} p_{2 \nu} u\left(\vec{p}_{1}, \lambda_{1}\right) I F_{N^{*} s}(I) .
$$

$N^{*}\left(D_{13}\right)$ pole diagram:

$$
\kappa \frac{f_{N^{*} N \eta}^{2}}{m_{\pi}^{4}} \bar{u}\left(\vec{p}_{3}, \lambda_{3}\right) \gamma^{5} p_{4} p_{4 \mu} \frac{1}{2 m_{N^{*}}^{0}} \frac{P^{\mu \nu}(q)}{E-m_{N^{*}}^{0}} \gamma^{5} p_{2} p_{2 \nu} u\left(\vec{p}_{1}, \lambda_{1}\right) I F_{N^{*} s}(I),
$$

$I F_{N^{*}{ }^{*}}(1 / 2)=1$.

\section{6. $\eta N \rightarrow \rho N$}

$N^{*}\left(S_{11}\right)$ pole diagram:

$$
-i \kappa \frac{f_{N^{*} N \eta} g_{N^{*} N \rho}}{m_{\pi}} \bar{u}\left(\vec{p}_{3}, \lambda_{3}\right) \gamma^{5} \xi^{*}\left(\vec{p}_{4}, \lambda_{4}\right) \frac{1}{2 m_{N^{*}}^{0}} \frac{q+m_{N^{*}}^{0}}{E-m_{N^{*}}^{0}} p_{2} u\left(\vec{p}_{1}, \lambda_{1}\right) I F_{N^{*} s}(I) .
$$

$N^{*}\left(D_{13}\right)$ pole diagram:

$$
i \kappa \frac{f_{N^{*} N \eta} f_{N^{*} N \rho}}{m_{\pi}^{2} m_{\rho}} \bar{u}\left(\vec{p}_{3}, \lambda_{3}\right)\left(p_{4} \epsilon_{\mu}^{*}\left(\vec{p}_{4}, \lambda_{4}\right)-p_{4 \mu} k^{*}\left(\vec{p}_{4}, \lambda_{4}\right)\right) \frac{P^{\mu \nu}(q)}{2 m_{N^{*}}\left(E-m_{N^{*}}^{0}\right)} p_{2} \gamma_{\nu}^{5} p_{2} u\left(\vec{p}_{1}, \lambda_{1}\right) I F_{N^{*} s}(I),
$$

$I F_{N^{*}{ }_{S}}(1 / 2)=\sqrt{3}$

$$
\text { 7. } \eta N \rightarrow \pi \Delta
$$

$N^{*}\left(S_{11}\right)$ pole diagram:

$$
\kappa \frac{f_{N^{*} N \eta} f_{N^{*} \Delta \pi}}{m_{\pi}^{2}} \bar{u}_{\mu}\left(\vec{p}_{3}, \lambda_{3}\right) p_{4}^{\mu} \gamma^{5} \frac{1}{2 m_{N^{*}}^{0}} \frac{q+m_{N^{*}}^{0}}{E-m_{N^{*}}^{0}} p_{2} u\left(\vec{p}_{1}, \lambda_{1}\right) I F_{N^{*} s}(I)
$$

$N^{*}\left(P_{13}\right)$ pole diagram:

$$
\kappa \frac{f_{N^{*} N \eta} f_{N^{*} \Delta \pi}}{m_{\pi}^{2}} \bar{u}_{\mu}\left(\vec{p}_{3}, \lambda_{3}\right) \gamma^{5} p_{4} \frac{1}{2 m_{N^{*}}^{0}} \frac{P^{\mu \nu}(q)}{E-m_{N^{*}}^{0}} p_{2 \nu} u\left(\vec{p}_{1}, \lambda_{1}\right) I F_{N^{*} s}(I)
$$

$N^{*}\left(D_{13}\right)$ pole diagram:

$$
-\kappa \frac{f_{N^{*} N \eta} f_{N^{*} \Delta \pi}}{m_{\pi}^{3}} \bar{u}_{\mu}\left(\vec{p}_{3}, \lambda_{3}\right) p_{4} \frac{1}{2 m_{N^{*}}^{0}} \frac{P^{\mu \nu}(q)}{E-m_{N^{*}}^{0}} \gamma^{5} p_{2} p_{2 \nu} u\left(\vec{p}_{1}, \lambda_{1}\right) I F_{N^{*} s}(I),
$$

$I F_{N^{*}{ }^{*}}(1 / 2)=-\sqrt{2}$.

\section{8. $\rho N \rightarrow \rho N$}

$N^{*}\left(S_{11}\right)$ pole diagram:

$$
\kappa g_{N^{*} N \rho}^{2} \bar{u}\left(\vec{p}_{3}, \lambda_{3}\right) \gamma^{5} \xi^{*}\left(\vec{p}_{4}, \lambda_{4}\right) \frac{1}{2 m_{N^{*}}^{0}} \frac{\phi+m_{N^{*}}^{0}}{E-m_{N^{*}}^{0}} \gamma^{5} \xi\left(\vec{p}_{2}, \lambda_{2}\right) u\left(\vec{p}_{1}, \lambda_{1}\right) I F_{N^{*} s}(I) .
$$

$N^{*}\left(D_{13}, D_{33}\right)$ pole diagrams: 


$$
\kappa \frac{f_{N^{*} N \rho}^{2}}{m_{\rho}^{2}} \bar{u}\left(\vec{p}_{3}, \lambda_{3}\right)\left[p_{4} \epsilon^{*}\left(\vec{p}_{4}, \lambda_{4}\right)-p_{4 \mu} \epsilon^{*}\left(\vec{p}_{4}, \lambda_{4}\right)\right] \frac{P^{\mu \nu}(q)}{2 m_{N^{*}}\left(E-m_{N^{*}}^{0}\right)}\left[p_{2} \epsilon_{\nu}^{*}\left(\vec{p}_{2}, \lambda_{2}\right)-p_{2 \nu} \epsilon^{*}\left(\vec{p}_{2}, \lambda_{2}\right)\right] u\left(\vec{p}_{1}, \lambda_{1}\right) I F_{N^{*} s}(I),
$$

$I F_{N^{*} s}(1 / 2)=3, I F_{N^{*} s}(3 / 2)=1$.

$$
\text { 9. } \rho N \rightarrow \pi \Delta
$$

$N^{*}\left(D_{13}, D_{33}\right)$ pole diagrams:

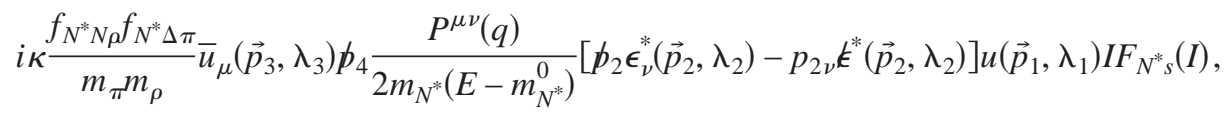

$I F_{N^{*} s}(1 / 2)=-\sqrt{6}, I F_{N^{*} s}(3 / 2)=\sqrt{\frac{5}{3}}$.

$$
\text { 10. } \pi \Delta \rightarrow \pi \Delta
$$

$N^{*}\left(S_{11}, S_{31}\right)$ pole diagrams:

$$
-\kappa \frac{f_{N^{*} \Delta \pi}^{2}}{m_{\pi}^{2}} \bar{u}_{\mu}\left(\vec{p}_{3}, \lambda_{3}\right) p_{4}^{\mu} \gamma^{5} \frac{1}{2 m_{N^{*}}^{0}} \frac{q+m_{N^{*}}^{0}}{E-m_{N^{*}}^{0}} \gamma^{5} p_{2}^{\nu} u_{\nu}\left(\vec{p}_{1}, \lambda_{1}\right) I F_{N^{*} s}(I)
$$

$N^{*}\left(P_{31}\right)$ pole diagram:

$$
\kappa \frac{f_{N^{*} \Delta \pi}^{2}}{m_{\pi}^{2}} \bar{u}_{\mu}\left(\vec{p}_{3}, \lambda_{3}\right) p_{4}^{\mu} \frac{1}{2 m_{N^{*}}^{0}} \frac{\phi+m_{N^{*}}^{0}}{E-m_{N^{*}}^{0}} p_{2}^{\nu} u_{\nu}\left(\vec{p}_{1}, \lambda_{1}\right) I F_{N^{*} s}(I)
$$

$N^{*}\left(P_{13}\right)$ pole diagram:

$$
\kappa \frac{f_{N^{*} \Delta \pi}^{2}}{m_{\pi}^{2}} \bar{u}_{\mu}\left(\vec{p}_{3}, \lambda_{3}\right) \gamma^{5} p_{4} \frac{1}{2 m_{N^{*}}^{0}} \frac{P^{\mu \nu}(q)}{E-m_{N^{*}}^{0}} \gamma^{5} p_{2} u_{\nu}\left(\vec{p}_{1}, \lambda_{1}\right) I F_{N^{*} s}(I)
$$

$N^{*}\left(D_{13}, D_{33}\right)$ pole diagrams:

$$
\kappa \frac{f_{N^{*} \Delta \pi}^{2}}{m_{\pi}^{2}} \bar{u}_{\mu}\left(\vec{p}_{3}, \lambda_{3}\right) p_{4} \frac{1}{2 m_{N^{*}}^{0}} \frac{P^{\mu \nu}(q)}{E-m_{N^{*}}^{0}} p_{2} u_{\nu}\left(\vec{p}_{1}, \lambda_{1}\right) I F_{N^{*} s}(I)
$$

$I F_{N^{*} s}(1 / 2)=2, I F_{N^{*} s}(3 / 2)=\frac{5}{3}$.

[1] R. Koch, Z. Phys. C 29, 597 (1985).

[2] R. A. Arndt, I. I. Strakovsky, R. L. Workman, and M. M. Pavan, Phys. Rev. C 52, 2120 (1995).

[3] R. A. Arndt, R. L. Workman, I. I. Strakovsky, and M. M. Pavan, nucl-th/9807087.

[4] M. Mojžiš, Eur. Phys. J. C 2, 181 (1998).

[5] N. Fettes and U. Meißner, Nucl. Phys. A693, 693 (2001).

[6] B. C. Pearce and B. K. Jennings, Nucl. Phys. A528, 655 (1991).

[7] F. Gross and Y. Surya, Phys. Rev. C 47, 703 (1993).

[8] O. Krehl, C. Hanhart, S. Krewald, and J. Speth, Phys. Rev. C 62, 025207 (2000).

[9] H. Machner and J. Haidenbauer, J. Phys. G 25, R231 (1999).

[10] P. Moskal, M. Wolke, A. Khoukaz, and W. Oelert, Prog. Part. Nucl. Phys. 49, 1 (2002).

[11] E. Hernández and E. Oset, Phys. Lett. B 350, 158 (1995).

[12] C. Hanhart, J. Haidenbauer, A. Reuber, C. Schütz, and J.
Speth, Phys. Lett. B 358, 21 (1995).

[13] C. Hanhart, J. Haidenbauer, O. Krehl, and J. Speth, Phys. Lett. B 444, 25 (1998).

[14] M. Batinić and A. Švarc, Phys. Scr. 56, 321 (1997).

[15] G. Fäldt and C. Wilkin, Phys. Scr. 64, 427 (2001).

[16] K. Nakayama, J. Speth, and T.-S. H. Lee, Phys. Rev. C 65 , 045210 (2002).

[17] V. Baru, A. M. Gasparyan, J. Haidenbauer, C. Hanhart, A. E. Kudryavtsev, and J. Speth, Phys. Rev. C 67, 024002 (2003).

[18] K. Nakayama, A. Szczurek, C. Hanhart, J. Haidenbauer, and J. Speth, Phys. Rev. C 57, 1580 (1998).

[19] K. Tsushima and K. Nakayama, nucl-th/0304017.

[20] A. Gasparian, J. Haidenbauer, C. Hanhart, L. Kondratyuk, and J. Speth, Phys. Lett. B 480, 273 (2000).

[21] R. Shyam, G. Penner, and U. Mosel, Phys. Rev. C 63, 022202 (2001).

[22] C. Schütz, J. W. Durso, K. Holinde, and J. Speth, Phys. Rev. C 
49, 2671 (1994).

[23] C. Schütz, K. Holinde, J. Speth, B. C. Pearce, and J. W. Durso, Phys. Rev. C 51, 1374 (1995).

[24] C. Schütz, J. Haidenbauer, J. W. Durso, and J. Speth, Phys. Rev. C 57, 1464 (1998).

[25] O. Krehl, C. Hanhart, S. Krewald, and J. Speth, Phys. Rev. C 60, 055206 (1999).

[26] A. B. Gridnev and N. G. Kozlenko, Eur. Phys. J. A 4, 187 (1999).

[27] G. Penner and U. Mosel, Phys. Rev. C 66, 055211 (2002).

[28] V. Shklyar, G. Penner, and U. Mosel, hep-th/0301152.

[29] K. Hagiwara et al., Phys. Rev. D 66, 010001 (2002).

[30] R. Aaron, R. D. Amado, and J. E. Young, Phys. Rev. 174, 2022 (1968).

[31] A. Müller-Groeling, K. Holinde, and J. Speth, Nucl. Phys. A513, 557 (1990).

[32] J. Wess and B. Zumino, Phys. Rev. 163, 1727 (1967).

[33] V. Bernard, N. Kaiser, and U.-G. Meissner, Nucl. Phys. A615, 483 (1997).

[34] K. Erkelenz, Phys. Rep. 13, 191 (1974).

[35] R. Aaron and R. D. Amado, Phys. Rev. 150, 857 (1966).

[36] R. T. Cahill and I. H. Sloan, Nucl. Phys. A165, 161 (1971).

[37] J. W. Durso, A. D. Jackson, and B. J. Verwest, Nucl. Phys. A345, 471 (1980).

[38] C. Caso et al., Eur. Phys. J. C 3, 1 (1998).

[39] A. Gasparyan, Ph.D. thesis, Forschungszentrum Jülich Report No. 40152002.

[40] S. Weinberg, Phys. Rev. Lett. 17, 616 (1966).

[41] Y. Tomozawa, Nuovo Cimento A 46, 707 (1966).

[42] G. Penner and U. Mosel, Phys. Rev. C 65, 055202 (2002).
[43] T. Inoue, E. Oset, and M. J. Vicente Vacas, Phys. Rev. C 65, 035204 (2002).

[44] C. Hanhart and K. Nakayama, Phys. Lett. B 454, 176 (1999).

[45] O. Krehl and J. Speth, Acta Phys. Pol. B 29, 2477 (1998).

[46] A. Sibirtsev, S. Schneider, C. Elster, J. Haidenbauer, S. Krewald, and J. Speth, Phys. Rev. C 65, 044007 (2002).

[47] V. Yu. Grishina, L. A. Kondratyuk, M. Büscher, J. Haidenbauer, C. Hanhart, and J. Speth, Phys. Lett. B 475, 9 (2000).

[48] H. Garcilazo and M. T. Peña, Phys. Rev. C 66, 034606 (2002).

[49] W. R. Frazer and J. R. Fulco, Phys. Rev. 117, 1603 (1960).

[50] R. Koch, Nucl. Phys. A448, 707 (1986).

[51] R. M. Brown et al., Nucl. Phys. B153, 89 (1979).

[52] F. Bulos et al., Phys. Rev. Lett. 13, 486 (1964).

[53] F. Bulos et al., Phys. Rev. 187, 1827 (1969).

[54] W. Deinet et al., Nucl. Phys. B11, 495 (1969).

[55] J. Feltesse et al., Nucl. Phys. B93, 242 (1975).

[56] B. W. Richards et al., Phys. Rev. D 1, 10 (1970).

[57] T. Morrison, Ph.D. thesis, The George Washington University, 1999.

[58] N. C. Debenham et al., Phys. Rev. D 12, 2545 (1975).

[59] G. Janßen, K. Holinde, and J. Speth, Phys. Rev. C 54, 2218 (1996).

[60] J. W. Durso, Phys. Lett. B 184, 348 (1987).

[61] C. Schütz,. Ph.D. thesis, Forschungszentrum Jülich Report, No. 3130, 1995.

[62] G. E. Brown and W. Weise, Phys. Rep. 22, 279 (1975).

[63] G. Janßen, B. C. Pearce, K. Holinde, and J. Speth, Phys. Rev. D 52, 2690 (1995).

[64] O. Krehl and J. Speth, Nucl. Phys. A623, 162c (1997).

[65] R. Koch and E. Pietarinen, Nucl. Phys. A336, 331 (1980). 